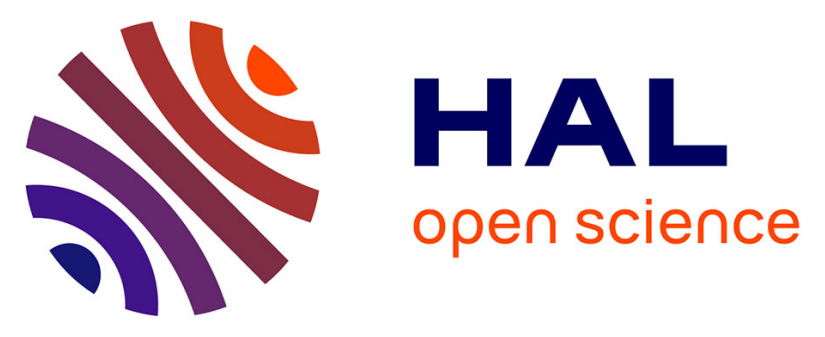

\title{
Tailoring the Morphology of Polymer/Montmorillonite Hybrid Latexes by Surfactant-Free Emulsion Polymerization Mediated by Amphipathic MacroRAFT Agents
}

Rodrigo Duarte Silva, Thaíssa de Camargo Chaparro, Igor Stefanichen Monteiro, Pierre-Yves Dugas, Franck D'agosto, Muriel Lansalot, Amilton Martins dos Santos, Elodie Bourgeat-Lami

\section{- To cite this version:}

Rodrigo Duarte Silva, Thaíssa de Camargo Chaparro, Igor Stefanichen Monteiro, Pierre-Yves Dugas, Franck D'agosto, et al.. Tailoring the Morphology of Polymer/Montmorillonite Hybrid Latexes by Surfactant-Free Emulsion Polymerization Mediated by Amphipathic MacroRAFT Agents. Macromolecules, 2019, 52 (13), pp.4979-4988. 10.1021/acs.macromol.9b00741 . hal-02325028

\section{HAL Id: hal-02325028 \\ https://hal.science/hal-02325028}

Submitted on 13 Nov 2020

HAL is a multi-disciplinary open access archive for the deposit and dissemination of scientific research documents, whether they are published or not. The documents may come from teaching and research institutions in France or abroad, or from public or private research centers.
L'archive ouverte pluridisciplinaire HAL, est destinée au dépôt et à la diffusion de documents scientifiques de niveau recherche, publiés ou non, émanant des établissements d'enseignement et de recherche français ou étrangers, des laboratoires publics ou privés. 


\section{Tailoring the Morphology of Polymer/Montmorillonite Hybrid}

\section{Latexes by Surfactant-Free Emulsion Polymerization Mediated}

\section{by Amphipathic MacroRAFT Agents}

Rodrigo Duarte Silva, ${ }^{\dagger}$ Thaíssa de Camargo Chaparro, ${ }^{\dagger,}$ Igor Stefanichen Monteiro, ${ }^{\dagger}$ PierreYves Dugas, ${ }^{\ddagger}$ Franck D’Agosto, ${ }^{\ddagger}$ Muriel Lansalot, ${ }^{\ddagger}$ Amilton Martins dos Santos, ${ }^{*}{ }^{\dagger}$ Elodie 'Bourgeat-Lami, *,

"Univ. Lyon, Université Claude Bernard Lyon 1, CPE Lyon, CNRS, UMR 5265, Chemistry, Catalysis, Polymers and Processes (C2P2), 43 Bvd. du 11 Novembre 1918, F-69616 Villeurbanne, France. ${ }^{\dagger}$ Engineering School of Lorena - University of Sao Paulo, Laboratory of Polymers, 12602-810 Lorena/SP, Brazil

*E-mail: bourgeat@ @ lcpp.cpe.fr (E. B-L.)

*E-mail: amsantos@usp.br (A. M. S.) 
FOR TABLE OF CONTENTS USE ONLY

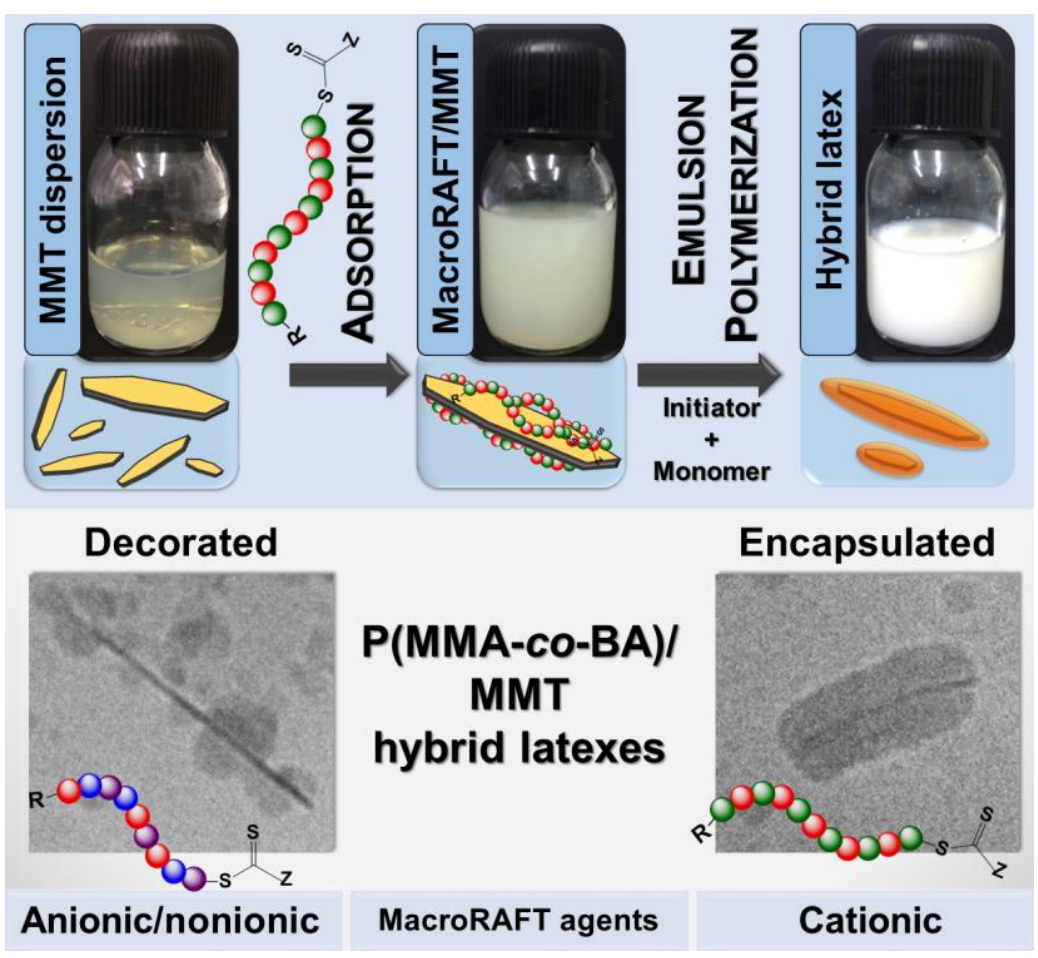




\begin{abstract}
In this work, we report the RAFT-mediated synthesis of polymer/Montmorillonite (MMT) hybrid latexes by surfactant-free emulsion polymerization. Macromolecular RAFT (macroRAFT) agents combining repeating units of acrylic acid (AA), n-butyl acrylate (BA), poly(ethylene glycol) methyl ether acrylate (PEGA) or 2-(dimethylamino)ethyl methacrylate (DMAEMA) were adsorbed on exfoliated sodium MMT platelets and the resulting aqueous colloidal dispersions were used as the reaction medium for emulsion copolymerization of methyl methacrylate and BA under starved-feed conditions. The AA- and PEGA-containing macroRAFT agents led to the formation of polymer decorated-clay platelets, indicating some affinity of the growing polymer chains for the clay surface, albeit not sufficient for the complete encapsulation of the clay sheets inside the polymer particles. When DMAEMAbased macroRAFT agents were used, TEM analyses of the hybrid latexes revealed the formation of a polymer layer on the MMT platelets indicating that the polymerization took place on the inorganic surface resulting in full encapsulation while preserving the shape anisotropy of the original clay mineral.
\end{abstract}




\section{INTRODUCTION}

Polymer/clay nanocomposites have become the focus of considerable attention of academic and industrial research as the incorporation of clay inside a polymer matrix often leads to materials with enhanced mechanical, thermal or barrier properties. ${ }^{1,2}$ While there is still a significant interest in the use of clays in polymeric materials to enhance physical and mechanical properties, a great research effort has recently been conducted towards the precise control of nanoparticle arrangement and distribution within the final composite material to modulate its properties. ${ }^{3,4}$ Among the variety of methods used to prepare polymer/clay nanocomposites, in situ heterophase polymerization in aqueous media is particularly interesting for environmental considerations. Besides, techniques such as emulsion or miniemulsion polymerizations allow obtaining polymer/clay latexes that may be potentially used to manufacture a variety of specialty products such as latex paints, adhesives or coatings. ${ }^{5}$ If the polymerization takes place at the surface of the clay mineral preserving the contours of the inorganic nanoparticles while the polymer chains grow, the clay platelets may be encapsulated inside a polymer shell, leading to anisotropic core-shell particles that could be arranged inside the nanocomposite film in a controlled way, which should directly influence the macroscopic performance of the final nanocomposite material.

Emulsion $^{5}$ and miniemulsion ${ }^{6}$ polymerizations have been successfully applied for the production of polymer/clay nanocomposite latexes. Small fillers such as Laponite clay platelets may be located at the polymer/water interface, in the so-called clay-armored morphology, ${ }^{7}$ or embedded inside the polymer particles forming polymer-encapsulated clay particles. $^{6}$ However, attempts to encapsulate high aspect ratio clay sheets such as Montmorillonite (MMT) by the same methods have revealed challenging. ${ }^{8}{ }^{9}$ Indeed, particles with complex morphologies are often obtained instead of the targeted encapsulated 
morphology. ${ }^{10,11}$ In addition, although there are a few works describing successful MMT encapsulation, ${ }^{12,13}$ they all require surfactant, which may negatively impact film properties, and is also not environmentally-friendly.

A few years ago, a new process that allows the preparation of self-stabilized composite particles in the absence of additional surfactant has been reported in the literature. ${ }^{14,15}$ In short, this process takes advantage of the recent developments in reversible-deactivation radical polymerization (RDRP) in aqueous dispersed media, and the so-called polymerization induced self-assembly of block copolymers, and uses living amphiphilic random copolymers, which are adsorbed on the inorganic particles to encourage the emulsion polymerization to occur at their surface. In most cases, these copolymers are synthesized via reversible addition-fragmentation chain transfer (RAFT) polymerization and thus possess an active chain end that can be reactivated for the polymerization of hydrophobic monomers. In addition, the relatively high hydrophilicity of these copolymers provides stability for the aqueous dispersion of the inorganic particles before the polymerization and also contributes to the stability of the final particles. This approach, which was pioneered by RAFT, has also been recently implemented using other RDRP techniques such as nitroxide-mediated polymerization $^{16,17,18}$ and atom transfer radical polymerization. ${ }^{19,20}$ Since the first report on the encapsulation of titanium dioxide particles by Nguyen et al., ${ }^{21}$ a wide variety of nanoparticles have been encapsulated using this strategy such as quantum dots, carbon nanotubes, cerium oxide, graphene oxide, silica, iron oxide and layered double hydroxides (LDHs). ${ }^{22-32}$ Yet, there are comparatively few works reporting the synthesis of polymer/clay hybrid latexes by this method, ${ }^{32-35}$ of which a single one concerns MMT. ${ }^{36}$ In that article, the authors claim the encapsulation of MMT platelets by RAFT-mediated emulsion polymerization using low molar mass macroRAFT agents composed of BA and quaternized 2-(dimethylamino)ethyl acrylate units. However, there is only one experiment reported in this 
article, making it difficult to get a full picture of the relevant parameters influencing particle morphology.

Herein, we report the surfactant-free synthesis of poly(methyl methacrylate-co- $n$-butyl acrylate) (P(MMA-co-BA))/MMT hybrid latex particles through RAFT-mediated emulsion polymerization. In order to better understand the factors governing particle morphology while securing a good colloidal stability, different types of amphipathic macroRAFT agents that can specifically interact with MMT were selected in this work. The effects of the nature of the macroRAFT agent and the polymerization conditions on particle morphology and latex stability were assessed and correlated with the adsorption behavior, and in particular with the free macroRAFT agent concentration.

\section{EXPERIMENTAL SECTION}

\subsection{Materials}

Water was deionized before use in a Polaris Ultraviolet Reverse Osmosis apparatus (Sppencer Scientific Equipamentos). The monomers: $n$-butyl acrylate (BA, 99.5\%, BASF), styrene (St, 99.5\%, BASF) and methyl methacrylate (MMA, 95.5\%, BASF), kindly donated by BASF, were distilled under reduced pressure. The initiators: 4,4'-azobis(4-cyanopentanoic acid) (ACPA, 98\%, Aldrich) and 2,2'-azobis(2-methylpropionamidine) dihydrochloride (AIBA, 97\%, Aldrich) were used as received. Montmorillonite Clay Cloisite ${ }^{\circledR} \mathrm{Na}^{+}$(MMT) (BYK Additives \& Instruments) was dispersed in water by magnetic stirring followed by sonication in an ultrasound bath for $60 \mathrm{~min}$ and centrifuged at a centrifugal acceleration of $500 \mathrm{~g}$ for $1 \mathrm{~h}$ using a centrifuge Allegra ${ }^{\circledR}$ 64R $\left(\right.$ Beckman Coulter $^{\circledR}$ ) to eliminate large aggregates, resulting in a $1.0 \mathrm{wt} \%$ solids content clear aqueous dispersion. Different types of 
macroRAFT agents were used in this work (Table 1). All of them were synthesized by RAFT polymerization in solution using 4-cyano-4-thiothiopropylsulfanyl pentanoic acid as RAFT agent. Their detailed synthesis and characterization, as well as the study of their adsorption on MMT have been previously reported. ${ }^{37}$

Table 1. Compositions of the macroRAFT agents used in this work.

\begin{tabular}{|c|c|c|}
\hline Nonionic & MR1 & $\mathrm{P}\left(\mathrm{PEGA}_{4}-c o-\mathrm{BA}_{3}\right)\left(M_{\mathrm{n}}=2770 \mathrm{~g} \mathrm{~mol}^{-1}\right)$ \\
\hline \multirow[t]{3}{*}{ Anionic } & MR2 & $\mathrm{P}\left(\mathrm{AA}_{4}-c o-\mathrm{PEGA}_{4}-c o-\mathrm{BA}_{4}\right)\left(M_{\mathrm{n}}=3100 \mathrm{~g} \mathrm{~mol}^{-1}\right)$ \\
\hline & MR3 & $\mathrm{P}\left(\mathrm{AA}_{9}-c o-\mathrm{PEGA}_{9}-c o-\mathrm{BA}_{9}\right)\left(M_{\mathrm{n}}=6230 \mathrm{~g} \mathrm{~mol}^{-1}\right)$ \\
\hline & MR4 & $\mathrm{PAA}_{41}-b-\mathrm{P}\left(\mathrm{PEGA}_{6}-c o-\mathrm{BA}_{4}\right)\left(M_{\mathrm{n}}=7320 \mathrm{~g} \mathrm{~mol}^{-1}\right)$ \\
\hline \multirow[t]{5}{*}{ Cationic } & MR5 & $\mathrm{P}\left(\mathrm{DMAEMA}_{10}-c o-\mathrm{BA}_{4}\right)\left(M_{\mathrm{n}}=2310 \mathrm{~g} \mathrm{~mol}^{-1}\right)$ \\
\hline & MR6 & $\mathrm{P}\left(\mathrm{DMAEMA}_{16}-c o-\mathrm{BA}_{15}\right)\left(M_{\mathrm{n}}=4710 \mathrm{~g} \mathrm{~mol}^{-1}\right)$ \\
\hline & MR7 & $\mathrm{P}\left(\mathrm{DMAEMA}_{26}-c o-\mathrm{BA}_{20}\right)\left(M_{\mathrm{n}}=6950 \mathrm{~g} \mathrm{~mol}^{-1}\right)$ \\
\hline & MR8 & $\mathrm{P}\left(\mathrm{DMAEMA}_{17^{-}} c o-\mathrm{PEGA}_{7}-c o-\mathrm{BA}_{19}\right)\left(M_{\mathrm{n}}=8710 \mathrm{~g} \mathrm{~mol}^{-1}\right)$ \\
\hline & \multicolumn{2}{|r|}{ plus the quaternized analogues of MR5 and MR6: qMR5 and qMR6, respectively } \\
\hline
\end{tabular}

\subsection{Synthesis of Hybrid Latexes}

Hybrid latexes were prepared by surfactant-free starved-feed emulsion polymerization. In a typical experiment (R01, Table 2), the anionic macroRAFT agent (MR3, $0.25 \mathrm{~g}$ ) was firstly dissolved in deionized water (10.0 g) with magnetic stirring. Then, a certain amount of the MMT stock dispersion $\left(10.0 \mathrm{~g} \mathrm{~L}^{-1}, 10.5 \mathrm{~g}\right)$ was slowly dropped in the macroRAFT agent solution maintaining the stirring throughout. After the complete addition of MMT dispersion, the $\mathrm{pH}$ of the medium was adjusted to 8 with sodium hydroxide ( $0.5 \mathrm{M}$ aqueous solution). It was then introduced in a $50 \mathrm{~mL}$ three-necked round-bottom flask fitted with a condenser, a gas inlet and a magnetic stirrer. The initiator (ACPA, $0.0035 \mathrm{~g}$ ) was dissolved into water (1.5 $\mathrm{mL}$ ) and the solution was introduced in the reactor under stirring. The final dispersion was purged with nitrogen for $30 \mathrm{~min}$. The reaction was started upon immersing the flask in a 
heated oil bath at $70{ }^{\circ} \mathrm{C}$. At the same time, a deoxygenated monomer mixture $(\mathrm{MMA} / \mathrm{BA}=$ 10/1 weight ratio, $2.4 \mathrm{~g})$ was fed into the reactor at a constant flow rate $\left(0.6 \mathrm{~mL} \mathrm{~h}^{-1}\right)$ by means of a syringe pump (Genie, Kent Scientific). 
Table 2. Experimental conditions and characteristics of $\mathrm{P}(\mathrm{MMA}-\mathrm{co}-\mathrm{BA}) / \mathrm{MMT}$ hybrid latexes synthesized using nonionic $\left(\mathrm{P}\left(\mathrm{PEGA}_{4}-c o-\mathrm{BA}_{3}\right)\right.$ :

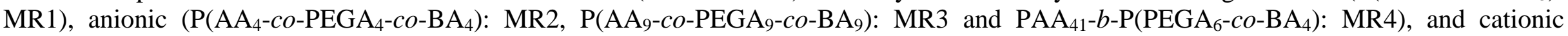
$\left(\mathrm{P}\left(\mathrm{DMAEMA}_{10}-c o-\mathrm{BA}_{4}\right): \mathrm{MR}\right.$, $\mathrm{P}\left(\mathrm{DMAEMA}_{16}-c o-\mathrm{BA}_{15}\right): \mathrm{MR}$, $\mathrm{P}\left(\mathrm{DMAEMA}_{26}-c o-\mathrm{BA}_{20}\right)$ : MR7 and P(DMAEMA $\left.{ }_{17}-c o-\mathrm{PEGA}_{7}-c o-\mathrm{BA}_{19}\right)$ : MR8) macroRAFT agents. ${ }^{a}$

\begin{tabular}{|c|c|c|c|c|c|c|c|c|c|c|c|}
\hline expt. & $\begin{array}{l}\text { macroRAFT } \\
(\text { MR) }\end{array}$ & $\begin{array}{c}\operatorname{mon}^{b} \\
(\mathrm{wt} \%)\end{array}$ & $\underset{(\mathbf{w t} / w t)}{\operatorname{MMA} / \mathbf{B A}}$ & $\mathbf{p H}$ & $\begin{array}{c}{[\mathrm{MR}]} \\
\left(\mathrm{mmol} \mathrm{L}^{-1}\right)\left(\mathrm{wt}_{\mathrm{mon}} \%\right)^{c}\end{array}$ & $\begin{array}{l}{[\mathrm{MR}]_{\text {Free }}{ }^{d}} \\
\left(\mathrm{mmol} \mathrm{L}^{-1}\right)\end{array}$ & $\begin{array}{c}{[\mathrm{MR}]_{\mathrm{Ads}}{ }^{d}} \\
\left(\mu \mathrm{mol} \mathrm{g}^{-1}\right)\left(\mathrm{mg} \mathrm{g}^{-1}\right)\end{array}$ & $\begin{array}{l}{[\mathrm{I}] /[\mathrm{MR}]^{e}} \\
(\mathrm{~mol} / \mathrm{mol})\end{array}$ & $\begin{array}{c}\operatorname{conv}_{(\%)}^{f} \\
\end{array}$ & $\begin{array}{l}Z_{\mathrm{av}, \mathbf{i}} / P d I_{\mathrm{i}}{ }^{g} \\
\quad(\mathbf{n m})\end{array}$ & $\begin{array}{l}Z_{\mathrm{av}, \mathrm{f}} / P d I_{\mathrm{f}}{ }^{h} \\
\quad(\mathrm{~nm})\end{array}$ \\
\hline R01 & MR3 & 10 & $10 / 1$ & 8 & $1.8(10.3)$ & 1.56 & $48.5(302)$ & 0.3 & 91 & $203 / 0.21$ & $138 / 0.48$ \\
\hline $\mathrm{R} 02$ & MR2 & 5 & $10 / 1$ & 8 & $3.6(10.3)$ & 3.20 & $80.3(250.8)$ & 0.3 & 82 & $215 / 0.24$ & $99 / 0.46$ \\
\hline $\mathrm{R} 03$ & MR3 & 5 & $10 / 1$ & 8 & $1.1(12.1)$ & 0.89 & $42.1(262.4)$ & 0.3 & 71 & $214 / 0.24$ & $113 / 0.45$ \\
\hline R04 & MR3 & 5 & $10 / 1$ & 5 & $1.1(12.1)$ & 0.65 & $90.4(563.3)$ & 0.3 & 59 & $217 / 0.23$ & $193 / 0.20$ \\
\hline R05 & MR4 & 10 & $10 / 1$ & 8 & $1.1(7.0)$ & 0.82 & $56.2(411.7)$ & 0.3 & 85 & $223 / 0.24$ & $97 / 0.28$ \\
\hline R06 & MR4 & 10 & $10 / 1$ & 5 & $1.1(7.0)$ & 0.62 & $95.8(701.2)$ & 0.3 & 70 & $223 / 0.23$ & $84 / 0.44$ \\
\hline R07 & MR1 + MR3 & 5 & $10 / 1$ & 5 & $2.6+2.4(42.5)$ & - & - & 0.3 & 77 & $209 / 0.22$ & $233 / 0.29$ \\
\hline R08 & MR6 & 10 & $10 / 1$ & 5 & $2.0(8.3)$ & 1.30 & $140.0(659.3)$ & 0.3 & 81 & $256 / 0.21$ & $259 / 0.22$ \\
\hline R09 & MR7 & 10 & $10 / 1$ & 5 & $1.7(10.4)$ & 1.20 & $100.2(696.1)$ & 0.3 & 84 & $234 / 0.20$ & $217 / 0.26$ \\
\hline $\mathrm{R} 10$ & MR8 & 10 & $10 / 1$ & 5 & $0.7(6.0)$ & 0.31 & $78.9(687.2)$ & 0.3 & 73 & $242 / 0.18$ & $205 / 0.22$ \\
\hline R11 & MR8 & 10 & $10 / 1$ & 5 & $0.7(6.0)$ & 0.31 & $78.9(687.2)$ & 1.0 & 78 & $251 / 0.27$ & $223 / 0.13$ \\
\hline $\mathrm{R} 12$ & MR8 & 10 & $10 / 1$ & 5 & $1.2(9.4)$ & 0.71 & $98.3(859.1)$ & 1.0 & 88 & $227 / 0.25$ & $222 / 0.13$ \\
\hline R13 & MR7 & 10 & $1 / 1$ & 5 & $1.7(10.4)$ & 1.20 & $100.2(696.1)$ & 0.3 & 95 & $218 / 0.19$ & $237 / 0.18$ \\
\hline $\mathrm{R} 14$ & MR8 & 10 & $1 / 1$ & 5 & $1.2(9.4)$ & 0.71 & $98.3(859.1)$ & 1.0 & 83 & $238 / 0.22$ & $259 / 0.13$ \\
\hline $\mathrm{R} 15^{i}$ & MR8 & 10 & $1.3 / 1^{j}$ & 5 & $1.4(11.1)$ & 0.87 & $105.5(919.3)$ & 0.3 & 89 & $241 / 0.24$ & $235 / 0.16$ \\
\hline $\mathrm{R} 16^{i}$ & MR8 & 10 & $1.5 / 1^{k}$ & 5 & $1.4(11.1)$ & 0.87 & $105.5(919.3)$ & 0.3 & 90 & $234 / 0.22$ & $245 / 0.13$ \\
\hline $\mathrm{R} 17^{i}$ & MR8 & 10 & $1.8 / 1^{l}$ & 5 & $1.4(11.1)$ & 0.87 & $105.5(919.3)$ & 0.3 & 84 & $239 / 0.21$ & $226 / 0.14$ \\
\hline $\mathrm{R} 18^{i}$ & MR8 & 10 & $1.8 / 1^{l}$ & 5 & $0.8(6.6)$ & - & - & 0.3 & 85 & $210 / 0.18$ & $178 / 0.19$ \\
\hline
\end{tabular}

${ }^{a} \mathrm{MMT}=5.0 \mathrm{~g} \mathrm{~L}^{-1}$ (except for R18, whose MMT $=3.0 \mathrm{~g} \mathrm{~L}^{-1}$ ); $T=70{ }^{\circ} \mathrm{C} .{ }^{b}$ Monomer content. ${ }^{c}$ Relative to monomer. ${ }^{d}$ Estimated from the adsorption isotherms. ${ }^{37 e}$ For experiments R01 to R07, Initiator (I) = ACPA; for experiments R08 to $14, \mathrm{I}=\mathrm{AIBA} .{ }^{f}$ Overall monomer conversion. Determined by DLS before $^{g}$ and after ${ }^{h}$ polymerization. ${ }^{i}$ Synthesized using a comonomer mixture with MMA/BA $=10 / 1 \mathrm{wt} / \mathrm{wt}\left(m_{1}\right)$ and another subsequent one with MMA/BA $=$ $1 / 1 \mathrm{wt} / \mathrm{wt}\left(m_{2}\right) .{ }^{j} m_{2} / m_{1}=5 \mathrm{wt} / \mathrm{wt} .{ }^{k} m_{2} / m_{1}=3 \mathrm{wt} / \mathrm{wt} .{ }^{l} m_{2} / m_{1}=2 \mathrm{wt} / \mathrm{wt}$. 
The final MMT concentration was $5.0 \mathrm{~g} \mathrm{~L}^{-1}$, based on the total water, and the final monomer concentration was $10 \mathrm{wt} \%$, based on the total mass, resulting in a MMT to monomer weight ratio of $\sim 5 \mathrm{wt} \%$. The reaction mixture was magnetically stirred under nitrogen flow and samples were withdrawn from the reactor with a syringe during the polymerization and immediately cooled to $0{ }^{\circ} \mathrm{C}$ to stop the reaction in order to follow the evolution of monomer conversion and of the hydrodynamic diameter with time. Once the monomer addition was complete, the reaction was allowed to proceed for 2 additional hours before it was stopped by immersing the reactor in an ice bath. A similar procedure was used for the synthesis of hybrid latexes with cationic macroRAFT agents (R08 to R18 in Table 2). However, in this case the macroRAFT agent/MMT dispersion was placed in an ice bath and sonicated in an ultrasonic processor Vibra CellTM VCX 750 equipped with a micro-tip (Sonics \& Materials Inc.) for 5 min at an amplitude of $30 \%$ before being introduced in the reactor. This procedure was necessary to redisperse the MMT platelets that tend to aggregate upon addition of the cationic macroRAFT agent and charge inversion. In addition, hydrochloric acid (0.5 M aqueous solution) was used to adjust the $\mathrm{pH}$ to 5 and AIBA was used as initiator. Experiments R15 to R18, conducted using the cationic macroRAFT agent MR8, were performed using a slightly modified procedure: two comonomer mixtures with different MMA/BA mass ratios were fed in the reactor, one after the other.

\subsection{Characterizations}

The monomer to polymer conversion was determined by gravimetric analysis. The mean particle size (mean hydrodynamic diameter, $Z_{\mathrm{av}}$ ) and the broadness of the particle size distribution (indicated by a dimensionless number called Polydispersity Index (PdI) - the higher this value, the broader the size distribution) were measured by dynamic light scattering 
(DLS) at $173^{\circ}$ using the Zetasizer Nano ZS instrument from Malvern. At least five measurements were performed for each sample. Cryo-TEM observations were carried out to prevent deformation/degradation of the soft latex particles due to drying and/or radiation damage at room temperature. The diluted samples were dropped onto 300 Mesh holey carbon films (Quantifoil R2/1) and quench-frozen in liquid ethane using a cryo-plunge workstation (made at LPS Orsay) as reported elsewhere. ${ }^{38}$ The specimens were then mounted on a precooled Gatan 626 specimen holder, transferred in the microscope (Phillips CM120) and observed at an accelerating voltage of $120 \mathrm{kV}$ (Centre Technologique des Microstructures $(\mathrm{CT} \mu)$, platform of the Claude Bernard 1 University, Villeurbanne, France).

\section{RESULTS AND DISCUSSION}

MMT is a phyllosilicate belonging to the smectite group of $2: 1$ clay minerals. ${ }^{39}$ MMT platelets are about $1 \mathrm{~nm}$ thick and of irregular shape. Their lateral dimensions may vary from ca. $30 \mathrm{~nm}$ to a few microns, resulting in high aspect ratios. ${ }^{40}$ In this work, we dispersed the MMT platelets in water with ultrasound and then centrifugated the resulting dispersion prior use in order to eliminate the large aggregates (non-exfoliated particles) that would settle down a few days later as described in the previous section. A TEM image and the particle size distribution determined by DLS of a typical MMT stock suspension obtained by this method, are displayed in Figures S1 and S2, respectively (Supporting Information). The particle size distribution was not uniform $(\mathrm{PdI}=0.23)$. Most particles have an average diameter of around $200 \mathrm{~nm}$ and a small fraction of large particles $\left(Z_{\mathrm{av} .}>4 \mu \mathrm{m}\right)$ could also be identified. When dispersed in water, MMT platelets display a characteristic charge heterogeneity: their faces (basal surface) have permanent negative charges due to isomorphic substitutions while their edges, which contain silanol and aluminol groups, are positively charged at $\mathrm{pH}$ values lower 
than the point of zero charge and negatively charged above it. ${ }^{41}$ The zeta potential of the MMT dispersions prepared following the previously mentioned protocol was $-30 \mathrm{mV}$, measured in the natural $\mathrm{pH}$ of the dispersions $(\sim 9.5) .{ }^{37}$ In this work, anionic, nonionic and cationic macroRAFT agents were evaluated for the synthesis of hybrid latexes (Table 1). In a previous study, we have shown that all of them are able to interact with the clay surface. ${ }^{37}$ In addition, they all contain hydrophobic BA units with the aim to favor the growth of polymer chains at the solid-liquid interface during emulsion polymerization. ${ }^{14,15}$ In MR4, these units are randomly distributed in one block while for the other macroRAFT agents, they are randomly distributed along the entire copolymer chains. This was done with the intent to minimize their self-assembly in the aqueous phase at the beginning of the polymerization. Thus, it is expected that the locus and potentially the control of the polymerization will be located at the surface of the MMT platelets. Although the molar masses of the copolymers synthesized by emulsion polymerization have not been measured, relatively low initiator-tomacroRAFT agent molar ratios have been used in most experiments so polymerizations could proceed under RAFT control. As the macroRAFT agents are intended to stabilize the polymer/MMT hybrid particles, copolymers with different molar masses have also been investigated to study the effect of this parameter, beyond composition, charge (negative, positive or neutral) as well as other experimental conditions, on latex stability and particle morphology.

\subsection{Hybrid Latexes Synthesized with Anionic MacroRAFT Agents}

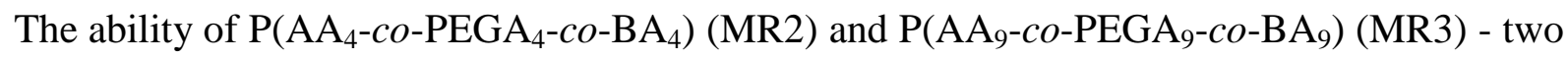
anionic macroRAFT agents of the same composition but having different molar masses - to form stable polymer/MMT hybrid latexes by starved-feed emulsion copolymerization of MMA and BA (10/1 weight ratio) in the presence of MMT $\left(5 \mathrm{~g} \mathrm{~L}^{-1}\right)$, was first evaluated at $\mathrm{pH}$ 
8 (R01 to R03 in Table 2) using ACPA as initiator. At this pH value, the PEGA units of the macroRAFT agent can interact with the MMT surface ${ }^{37}$ while the AA units are fully ionized maximizing their stabilizing potential. For a total monomer concentration of $10 \mathrm{wt} \%$ and $\sim 5$ wt $\%$ of MMT relative to monomer, a stable latex could be prepared with $1.8 \mathrm{mmol} \mathrm{L}^{-1}$ of MR3 (R01, Table 2) while the latex prepared with $3.6 \mathrm{mmol} \mathrm{L}^{-1}$ of the shorter MR2 (experiment SR1, Table S2, Supporting Information) did not remain stable even though the mass concentration of MR2 was the same as that of MR3 (the concentrations of macroRAFT agents were chosen on the basis of the adsorption isotherms to guarantee the surface of MMT platelets was adequately covered). When the monomer concentration was lowered to $5 \mathrm{wt} \%$ while keeping the MMT and MR2 concentration constant, a stable hybrid latex could successfully be synthesized (R02, Table 2). A stable sample could also be prepared with 5 wt\% monomer and MR3 using a lower macroRAFT agent concentration $(\mathrm{R} 03,[\mathrm{MR} 3]=1.1$ mmol L $\mathrm{L}^{-1}$, Table 2), which may be particularly interesting to avoid the formation of free polymer particles in water as the lower the macroRAFT agent concentration, the lower the amount of free macroRAFT. Thus, it can be concluded that $\mathrm{P}_{\left(\mathrm{AA}_{9}-\text { co-PEGA }\right.}-$ - $\left.0-\mathrm{BA}_{9}\right)$

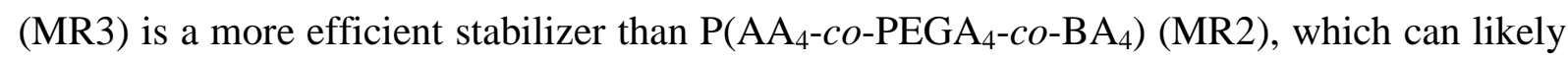
be attributed to its higher molar mass. Pearson et al., also reported that the macroRAFT length has a significant impact on latex stability during the synthesis of poly(methyl acrylate-co- $n$ butyl acrylate)/layered double hydroxide ( $\mathrm{LDH}$ ) composite particles using $\mathrm{P}(\mathrm{AA}-\mathrm{co}-\mathrm{BA})$ macroRAFT agents as stabilizers. ${ }^{34}$ Latexes R02 and R03 were analyzed by cryo-TEM (Figure 1), which revealed polymer-decorated MMT platelets consisting of individual spherical polymer particles distributed all along the clay basal surface (indicated by white arrows in Figure 1) and a great number of MMT-free polymer particles formed by secondary nucleation (bare MMT platelets could also be found in both samples, see Figure S3, Supporting Information). Indeed, as estimated from the adsorption isotherms and as displayed 
in Table 2, MMT-free macroRAFT agent is present in the aqueous phase at the beginning of the polymerizations. ${ }^{37}$ These polymer chains can undergo chain extension in water resulting in amphiphilic block copolymers capable of self-assembling.

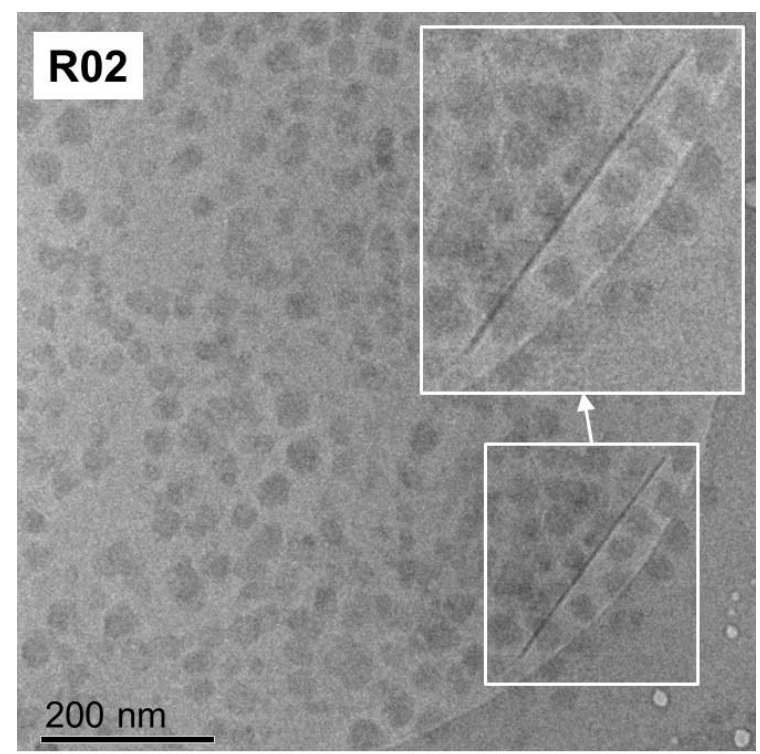

$\mathrm{P}\left(\mathrm{AA}_{4}\right.$-CO-PEGA $\mathrm{A}_{4}$-CO-BA $\left.{ }_{4}\right)$ $\mathrm{MR} 2, \mathrm{pH} 8$

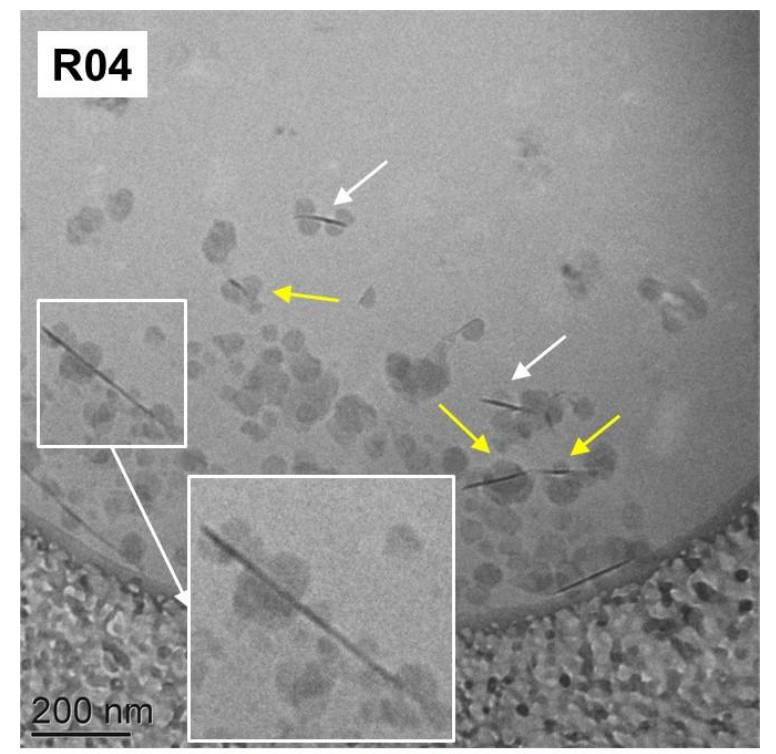

$\mathrm{P}\left(\mathrm{AA}_{9}\right.$-Co-PEGA $\left.\mathrm{A}_{9}-\mathrm{Co}-\mathrm{BA}_{9}\right)$

$\mathrm{MR} 3, \mathrm{pH} 5$
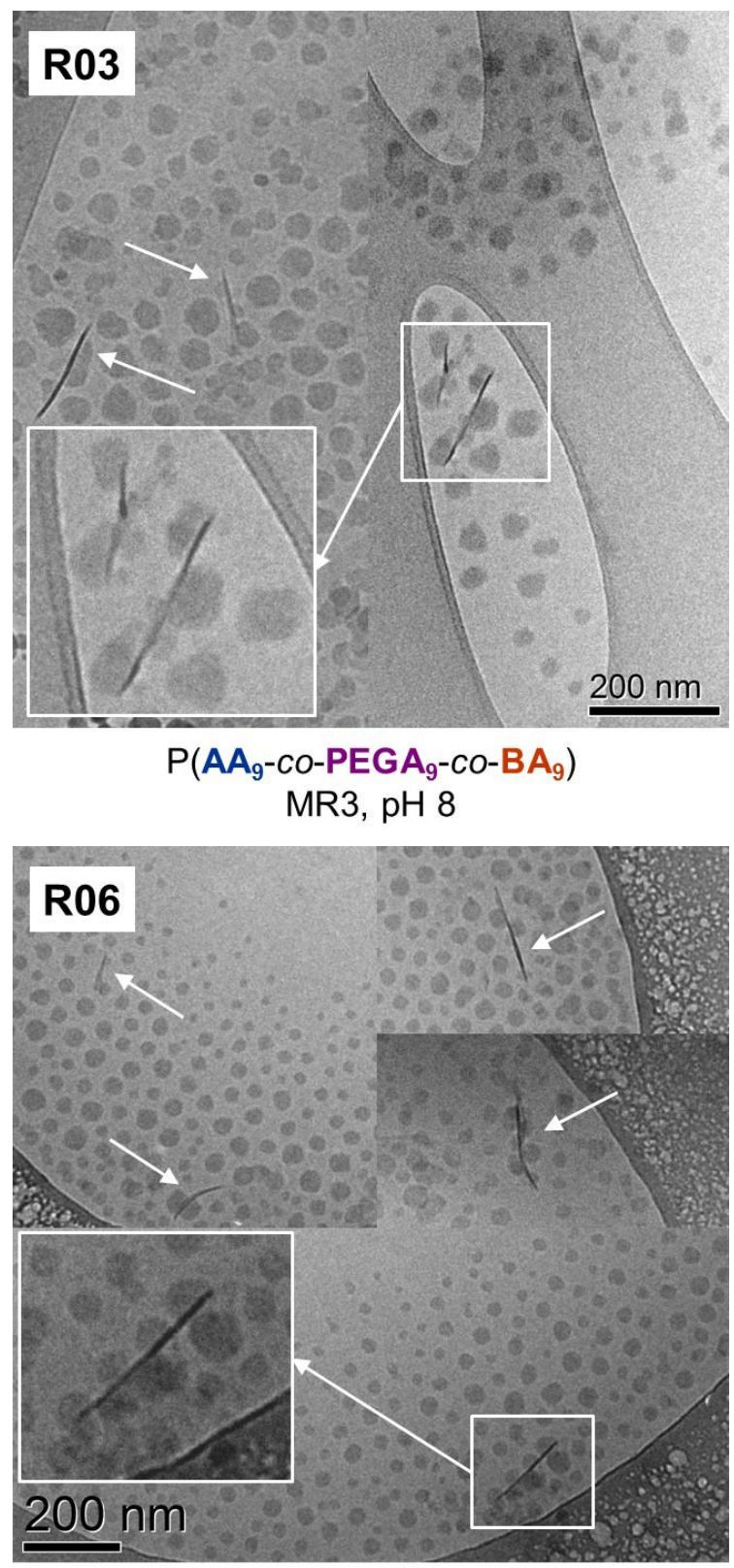

$\mathrm{PAA}_{41}-b-\mathrm{P}\left(\mathrm{PEGA}_{6}-\mathrm{CO}-\mathrm{BA}_{4}\right)$ $\mathrm{MR} 4, \mathrm{pH} 5$ 


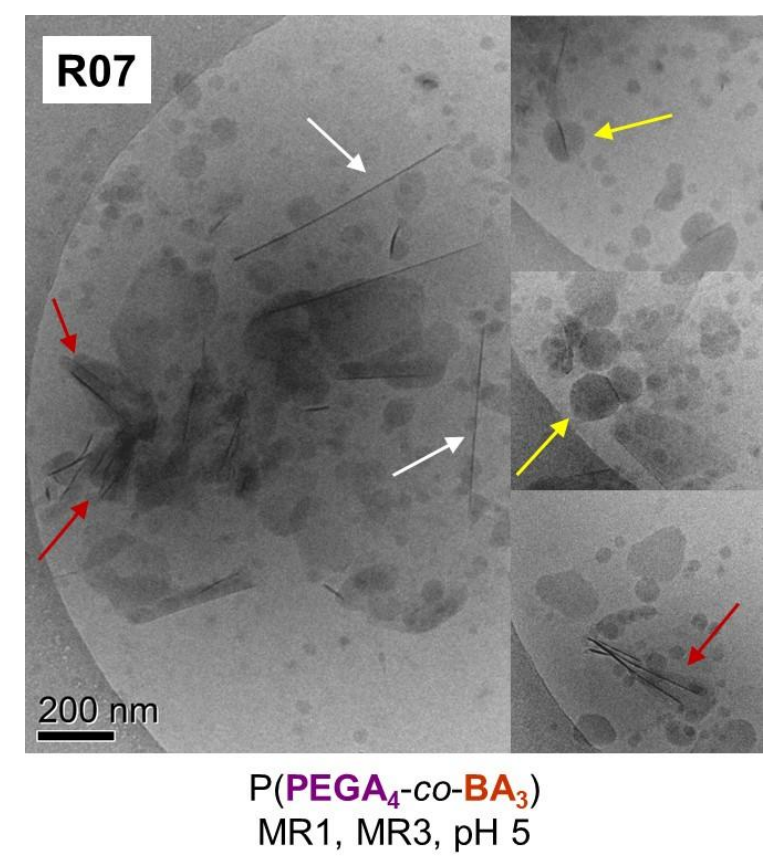

Figure 1. Cryo-TEM images of $\mathrm{P}(\mathrm{MMA}-\mathrm{co}-\mathrm{BA}) / \mathrm{MMT}$ hybrid latex particles prepared with MMA/BA $=10 / 1 \mathrm{wt} / \mathrm{wt}$ using pure anionic or a mixture of anionic and nonionic macroRAFT agents $\left(\right.$ MMT = $5.0 \mathrm{~g} \mathrm{~L}^{-1}$ ). R02 (MR2, pH 8), R03 (MR3, pH 8), R04 (MR3, pH 5), R06 (MR4, pH 5) and R07 (MR1 + MR3, pH 5). See Table 2 for experimental conditions. White arrows point to MMT platelets decorated with polymer particles, yellow arrows point to MMT platelets sandwiched between two polymer nodes and red arrows point to aggregated hybrid particles with MMT platelets almost fully covered with polymer.

The formation of secondary nucleated polymer particles resulted in a decrease of the average particle size and could also be followed by DLS (Figure 2A). It thus seems clear that the anionic macroRAFT agents are highly hydrophilic at $\mathrm{pH} 8$, which promotes stabilization but also favors the formation of discrete polymer nodes on the face of the MMT platelets together with secondary nucleated latex particles, instead of the targeted encapsulated morphology. 

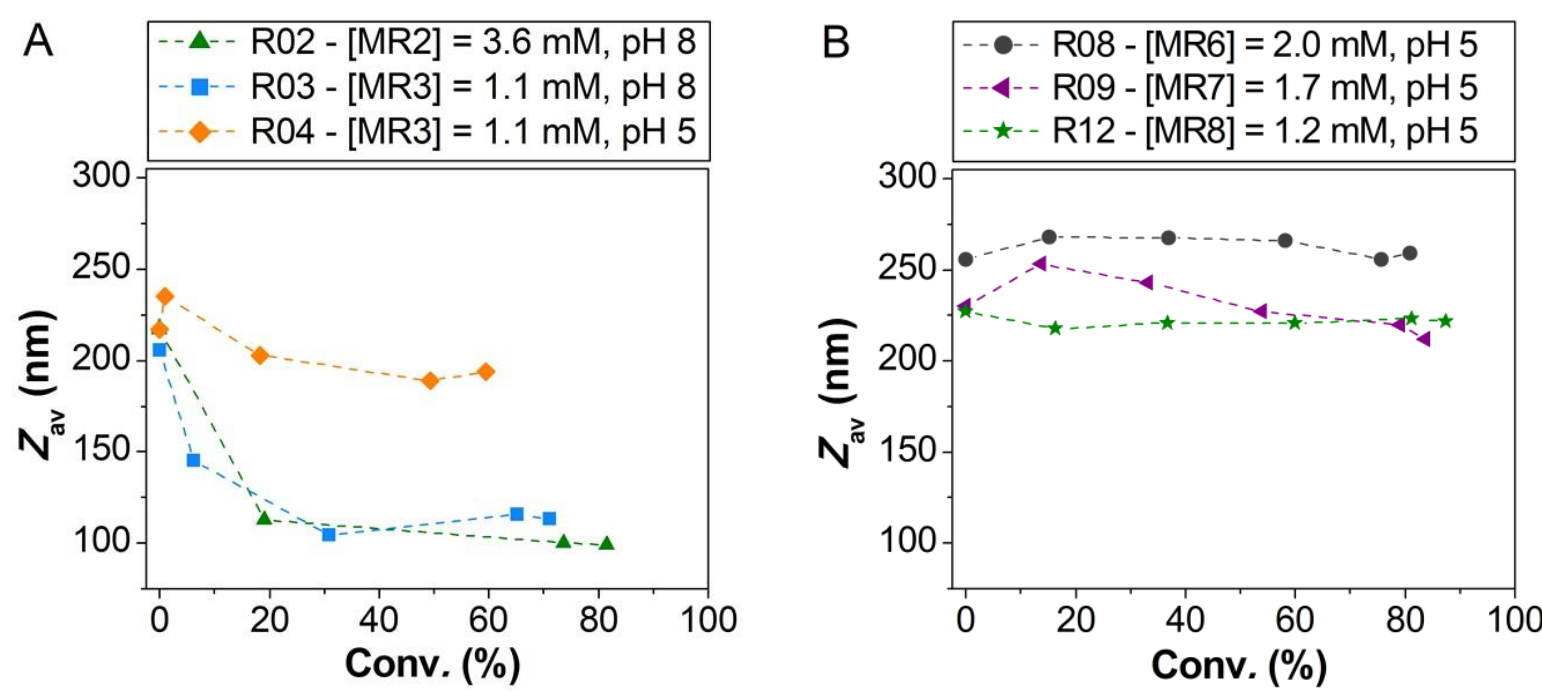

Figure 2. Evolution of particle size with conversion during the synthesis of

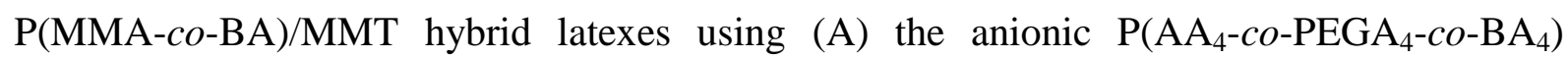

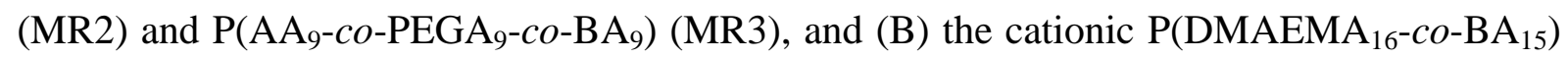
(MR6), $\mathrm{P}\left(\mathrm{DMAEMA}_{26}-\mathrm{co}-\mathrm{BA}_{20}\right)$ (MR7) and $\mathrm{P}\left(\mathrm{DMAEMA}_{17}-\mathrm{co}-\mathrm{PEGA}_{7}-\mathrm{co}-\mathrm{BA}_{19}\right)$ (MR8) macroRAFT agents. MMA/BA $=10 / 1 \mathrm{wt} / \mathrm{wt}$ and MMT $=5.0 \mathrm{~g} \mathrm{~L}^{-1}$. R02 (MR2, Monomer content $($ Mon $)=5 \mathrm{wt} \%$, green $\boldsymbol{\Delta})$, R03 (MR3, Mon = $5 \mathrm{wt} \%$, light blue $\boldsymbol{\pi})$, R04 (MR3, Mon $=5 \mathrm{wt} \%$, orange $\bullet$, R08 (MR6, Mon = $10 \mathrm{wt} \%$, black $\bullet)$, R09 (MR7, Mon = $10 \mathrm{wt} \%$, purple and R12 (MR8, Mon $=10 \mathrm{wt} \%$, green $\star)$. See Table 2 for detailed experimental conditions. The dotted lines are guides for the eyes. For the corresponding conversion with time plots, see Figure S4 in Supporting Information.

In order to render the MMT surface more compatible with the polymer phase, the $\mathrm{pH}$ of the reaction medium was lowered to 5. At this $\mathrm{pH}$ value, the affinity of MR2 and MR3 for the MMT surface was higher than at $\mathrm{pH} 8$ due to additional interaction between the AA units and the clay edges as well as to the lower ionization degree of MR2 and MR3, resulting in higher adsorbed amounts (and hence, less free macroRAFT agent in the aqueous phase, see Table 2) ${ }^{37}$ For these experiments, the monomer concentration was maintained at $5 \mathrm{wt} \%$ and the concentrations of MR2 and MR3 were, respectively, the same as those that afforded stable hybrid latexes in R02 and R03 experiments. The latex prepared with MR2 became unstable (SR2, Table S1), while a stable latex could yet be prepared with the longer MR3 (R04, Table 
2), confirming the higher effectiveness of this macroRAFT agent in stabilizing the hybrid latexes. The R04 sample was characterized by cryo-TEM and the images again showed polymer particles decorating the faces of the MMT platelets, but with polymer nodules closer to each other, and also MMT platelets sandwiched between polymer nodes (Figure 1). In this sample, the polymer phase seems to wet the inorganic surface to a better extent as the polymer particles are less spherical and tend to spread out on the MMT surface. In addition, fewer MMT-free polymer particles and no bare platelets were seen. Therefore, the reduction in the $\mathrm{pH}$ effectively resulted in an increase of the affinity between the polymer and the clay sheets, albeit not sufficient to ensure complete MMT encapsulation. This was also evidenced by DLS analysis as the final particle size of latex R04 was higher than that of latex R03 (Figure 2A), indicating that secondary nucleation was hindered at $\mathrm{pH}$. The analysis of Figure 1 revealed polymer particles in latex R04 with diameters as large as $83 \mathrm{~nm}$ while in latex R03 only polymer particles with diameters lower than $62 \mathrm{~nm}$ were found, in agreement with DLS analysis. These larger particles found in R04 could also be explained by the lower stabilization efficiency of MR3 at $\mathrm{pH}$ 5. When the $\mathrm{pH}$ was further lowered to 4 (SR3, Table S1), the latex prepared with MR3 lost stability. Thus, although polymer/clay compatibility could be increased by decreasing the $\mathrm{pH}$, the stability of the hybrid latexes was impaired. The macroRAFT agent $\mathrm{PAA}_{41}-b-\mathrm{P}\left(\mathrm{PEGA}_{6}-c o-\mathrm{BA}_{4}\right)(\mathrm{MR} 4)$ which contains a PAA block was next evaluated for the synthesis of $\mathrm{P}(\mathrm{MMA}-\mathrm{co}-\mathrm{BA}) / \mathrm{MMT}$ latexes. It was designed so the P(PEGA-co-BA) block would adsorb on the basal faces of the clay platelets while the PAA block would remain dangling in solution, resulting in a higher adsorbed mass at $\mathrm{pH} 8$ than that of MR3. ${ }^{37}$ As this macroRAFT agent contains more AA units, it is also expected to be a better stabilizer while promoting good polymer/clay compatibility as revealed by its adsorption behavior. It was thus employed in the synthesis of hybrid latexes with a monomer concentration of $10 \mathrm{wt} \%$ at $\mathrm{pH} 8$ and $\mathrm{pH} 5$ (R05 and R06, respectively, Table 2). Both latexes 
prepared with MR4 were stable. The morphology of the hybrid particles of latex R06 (pH 5) was however similar to the ones previously discussed (Figure 1). It nevertheless presented much more free polymer particles than latex R04, which was also prepared at $\mathrm{pH} 5$. The analysis of Figure 1 revealed free polymer particles with diameters as large as $42 \mathrm{~nm}$, which is lower than that of the large particles found in R04. This evidences the higher stabilization potential of this macroRAFT agent as latex R06 was prepared with $10 \mathrm{wt} \%$ of monomer at $\mathrm{pH}$ 5.

\subsection{Hybrid Latexes Synthesized with the Nonionic MacroRAFT Agent}

$\mathrm{P}\left(\mathrm{PEGA}_{4}-\mathrm{co}-\mathrm{BA}_{3}\right)(\mathrm{MR} 1)$, which is more hydrophobic, displays a stronger affinity for the MMT surface than the anionic macroRAFTs, ${ }^{37}$ and was therefore used in an attempt to more efficiently locate the polymerization at the surface of the clay platelets. When MR1 was used alone at $2.1 \mathrm{mmol} \mathrm{L}^{-1}$ with $5 \mathrm{wt} \%$ of monomer, the latex lost stability during polymerization (SR4, Table S1, Supporting Information), which may be due to the absence of ionizable units in its structure for stabilization of the hybrid particles. Thus, MR1 was used in conjunction with the anionic MR3 at pH 5 (R07, Table 2) keeping a total concentration of $5.0 \mathrm{mmol} \mathrm{L}{ }^{-1}$. According to the adsorption isotherm, $2.1 \mathrm{mmol} \mathrm{L}^{-1}$ of MR1 was sufficient to saturate the surface of the MMT platelets, while the additional amount of anionic macroRAFT agent (which was added later to the MMT dispersion) would guarantee stability. A stable latex was effectively obtained. Cryo-TEM showed that the polymer wet the faces of the MMT platelets more efficiently. Some hybrid particles (pointed by red arrows in Figure 1) even seem to be almost fully covered with polymer that completely spread out on the clay basal surface. Particles with "sandwich" morphology (pointed by yellow arrows) were also seen. These particles appear to display a larger polymer/inorganic interface than for the previous samples, suggesting that the polymer/inorganic compatibility was further improved. Although the 
desired full encapsulation of MMT platelets was not achieved, the use of a more hydrophobic macroRAFT agent led to the formation of hybrid particles with a relatively higher polymer coverage.

\subsection{Hybrid Latexes Synthesized with Cationic MacroRAFT Agents}

In the previous section, we have demonstrated that stable polymer/clay hybrid latexes could be prepared using AA- and/or PEGA-based amphipathic macroRAFT agents with different compositions and structures. Polymer/MMT compatibility could be improved by appropriately choosing the macroRAFT agent and the experimental conditions resulting in hybrid particles with morphologies going from a few polymer nodes decorating the MMT platelets to hybrid particles with polymer domains wetting the MMT basal surface to a greater extent. With the intent of encapsulating the MMT sheets, DMAEMA-based macroRAFT agents were lastly investigated. At $\mathrm{pH} 5$, these copolymers are positively charged, and can thus electrostatically interact with the clay platelets, leading to significantly higher adsorbed amounts when compared to the anionic or nonionic copolymers. Electrophoretic mobility measurements of the MMT platelets in the presence of increasing macroRAFT agent concentrations have shown that the clay undergoes charge inversion. ${ }^{37}$ At low concentrations, the macroRAFT agent is mostly located on the clay basal surface. Colloidal stability is lost as the concentration increases and zeta potential of MMT/macroRAFT agent complexes approaches zero. For relatively higher concentrations, the particles can be redispersed leading to stable complexes and free macroRAFT agent present in the aqueous phase. Similar results were obtained by Pavlovic et al. ${ }^{42}$ upon adsorption of anionic macroRAFT agents onto LDH clay platelets.

The first cationic macroRAFT agent investigated was $\mathrm{P}\left(\mathrm{qDMAEMA} \mathrm{A0}_{10}-\mathrm{co}-\mathrm{BA}_{4}\right)$ (qMR5). However, the latex synthesized with $2.0 \mathrm{mmol} \mathrm{L}^{-1}$ of qMR5 (which is the lowest 
concentration that afforded a stable MMT/qMR5 dispersion) $)^{37}$ and $5 \mathrm{wt} \%$ of monomer, lost stability at the beginning of the synthesis (SR5 in Table S2). The concentration was increased to $5.0 \mathrm{mmol} \mathrm{L}^{-1}$, but the latex was still unstable (SR6, Table S2). The longer P(qDMAEMA ${ }_{16}^{-}$ $c o$-BA 15$)$ macroRAFT agent (qMR6) $\left(2.0 \mathrm{mmol} \mathrm{L}^{-1}, \mathrm{SR} 7\right.$, Table S2) was also unsuccessful. In contrast, the same concentration of the non-quaternized $\mathrm{P}\left(\mathrm{DMAEMA}_{16}-\mathrm{Co}-\mathrm{BA}_{15}\right)$ (MR6) allowed the preparation of a stable latex at $\mathrm{pH} 5$ with $10 \mathrm{wt} \%$ monomer (R08, Table 2) despite the presence of a low amount of fine coagulum (see Figure S5, Supporting Information). The polymerization achieved a relatively high conversion (above 80\%) and the particle size remained nearly constant throughout the synthesis (Figure 2B). These results show that the non-quaternized DMAEMA-based macroRAFT agent is a better stabilizing precursor for the preparation of the hybrid latexes than its quaternized homologue, which may be related to the conformation of the adsorbed chains on the MMT platelets. Indeed, due to the stronger interaction of qMR6 with $\mathrm{MMT},{ }^{37}$ the chains of the quaternized macroRAFT agent are expected to adopt a flatter conformation on the clay surface than their nonquaternized counterparts, resulting in fewer segments dangling into solution, which would compromise the stabilization of the particles. Cryo-TEM of the R08 experiment (MR6) shows that, contrary to what has been previously observed for the anionic or non-ionic macroRAFT agents, a polymer layer was formed at the surface of the MMT platelets (pointed by white arrows in Figure 3). MMT-free polymer particles could also be seen, indicating that the polymerization took place both on the clay basal surface and in the water phase due to the presence of free macroRAFT agent at the beginning of the polymerization. With the intent to further improve colloidal stability, the concentration of MR6 was raised to $2.5 \mathrm{mmol} \mathrm{L}^{-1}$ (SR8, Table S2, Supporting Information). However, the particle size continuously increased during the polymerization until the latex lost stability (associated with very high PdIs) (Figures S3). Similar results were reported in the literature, ${ }^{29}, 34$ and attributed to the 
engagement of the free macroRAFT agent in the formation of stable polymer particles in the aqueous phase, no longer being available for the stabilization of the hybrid particles that would coagulate to some extent as the hydrophobic polymer layer was being formed.

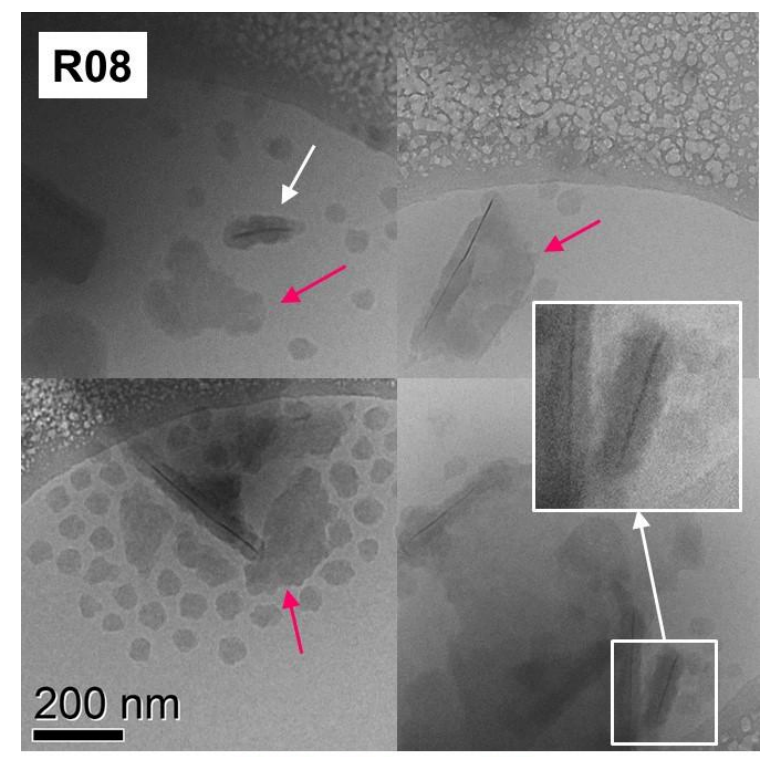

P(DMAEMA (D6 $\left._{16}-\mathrm{COA}_{15}\right)$

MR6, $\mathrm{pH} 5$

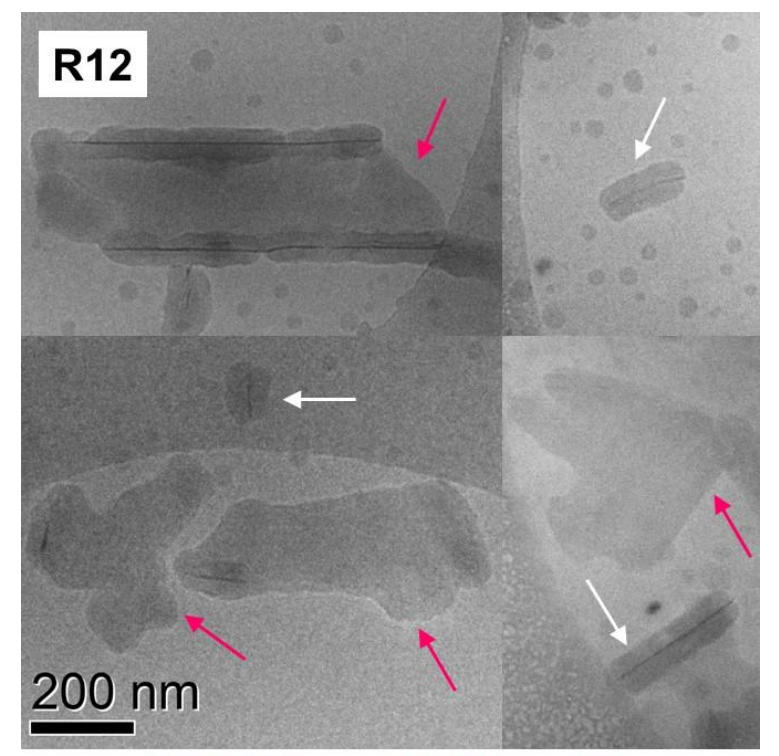

P(DMAEMA 17 -Co-PEGA - -Co-BA 19 )

$\mathrm{MR} 8, \mathrm{pH} 5$

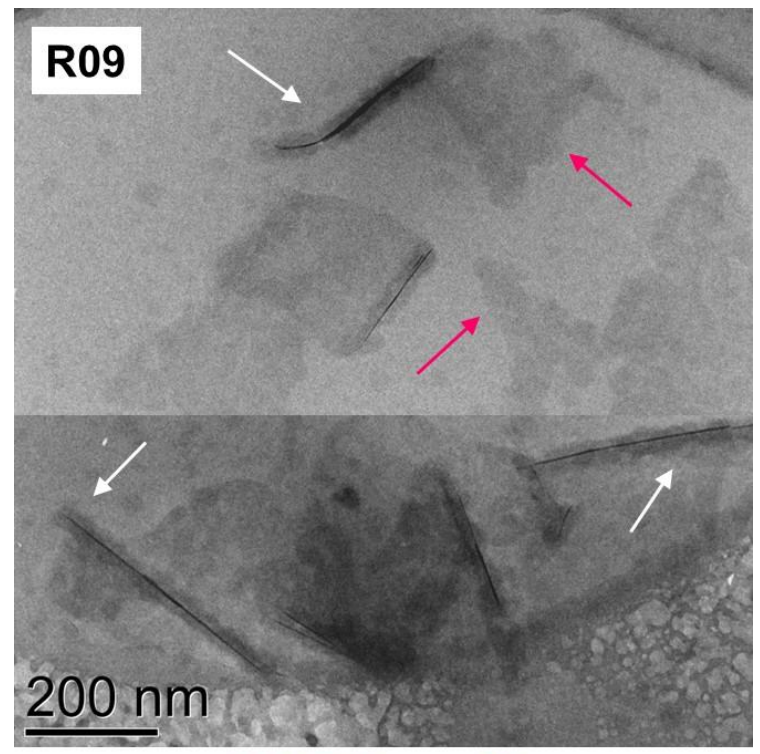

P(DMAEMA 26 $_{26}$-CO-BA 20

MR7, $\mathrm{pH} 5$

Figure 3. Cryo-TEM images of $\mathrm{P}(\mathrm{MMA}-\mathrm{co}-\mathrm{BA}) / \mathrm{MMT}$ hybrid latex particles prepared with MMA/BA $=10 / 1 \mathrm{wt} / \mathrm{wt}$ using cationic macroRAFT agents at $\mathrm{pH} 5$ (Monomer content (Mon) $=10 \mathrm{wt} \%$, MMT = $\left.5.0 \mathrm{~g} \mathrm{~L}^{-1}\right)$. R08 (MR6), R09 (MR7) and R12 (MR8). See Table 2 for experimental conditions. White arrows point to hybrid particles parallel to electron beam and pink arrows point to hybrid particles orthogonal to electron beam. 
Based on the results above for the anionic macroRAFT agents, a higher molar mass copolymer, $\mathrm{P}\left(\mathrm{DMAEMA}_{26}-\mathrm{co}-\mathrm{BA}_{20}\right)$ (MR7), was logically employed as an alternative strategy to improve latex stability. This macroRAFT is expected to adsorb on the MMT basal surface in such a conformation that allows more segments to be extended into solution, which should enhance stability. ${ }^{37}$ The latex prepared with $1.7 \mathrm{mmol} \mathrm{L}{ }^{-1}$ of $\mathrm{MR} 7$ at $\mathrm{pH} 5$ and a monomer concentration of $10 \mathrm{wt} \%$ (R09, Table 2) was indeed stable with no coagulum (Figure S7, Supporting Information). High monomer conversions were achieved (84\%) and particle size remained approximately constant during the polymerization (Figures 2B). The cryo-TEM images of Figure 3 revealed the formation of polymer-encapsulated MMT platelets, confirming that this macroRAFT agent was efficient in locating the polymerization at the clay surface.

Finally, a last macroRAFT agent was employed, P(DMAEMA $\left.17-c o-\mathrm{PEGA}_{7}-c o-\mathrm{BA}_{19}\right)(\mathrm{MR} 8)$. It has the highest molar mass of the series and contains PEO side chains, which were included to provide additional steric stabilization. Indeed, MR8 was the most effective cationic macroRAFT agent in dispersing the MMT platelets as a relatively lower amount of this macroRAFT agent was needed to yield MMT/macroRAFT agent complexes with particle sizes comparable to the size of bare MMT particles. ${ }^{37}$ The first hybrid latex was synthesized with $0.7 \mathrm{mmol} \mathrm{L}^{-1}$ of MR8 (R10, Table 2), which was the lowest concentration that yielded stable MMT/MR8 complex particles. The corresponding concentration of free MR8 at the beginning of the reaction was $0.4 \mathrm{mmol} \mathrm{L}^{-1}$. The resulting hybrid latex was stable although it presented a small amount of very fine aggregates (Figure S7, Supporting Information). However, the polymerization achieved a lower conversion (73\%) in comparison to the ones carried out with the other cationic-based macroRAFT agents, probably due to the low MR8 concentration. The initiator to MR8 molar ratio was therefore raised to 1.0 (R11, Table 2), but 
the latex synthesized under this condition presented more aggregates than the previous one (Figure S7, Supporting Information). Thus, the concentration of MR8 was increased to 1.2 mmol $\mathrm{L}^{-1}$ for a fixed $[\mathrm{AIBA}] /[\mathrm{MR} 8]$ molar ratio of 1.0 (R12, Table 2), which enabled the formation of a stable latex (Figure S7) with 88\% conversion. Cryo-TEM analysis revealed that the MMT platelets were successfully encapsulated by a thin polymer layer following the contours of the particles (Figure 3). In comparison to the hybrid particles prepared with MR6 or MR7, the polymer layer seems to be more regular, which may be due to the higher adsorbed mass of MR8, which would have favored the polymerization on the clay surface.

\subsection{Film Forming Hybrid Latexes}

Both $\mathrm{P}\left(\mathrm{DMAEMA}_{26}-\mathrm{co}-\mathrm{BA}_{20}\right) \quad(\mathrm{MR} 7)$ and $\mathrm{P}\left(\mathrm{DMAEMA}_{17}-\mathrm{co}-\mathrm{PEGA}_{7}-\mathrm{co}-\mathrm{BA}_{19}\right)$ (MR8) afforded hybrid latexes with very good colloidal stability, composed of MMT platelets covered by a polymer shell. However, the latexes were prepared with a comonomer ratio of $\mathrm{MMA} / \mathrm{BA}=10 / 1 \mathrm{wt} / \mathrm{wt}$, which would yield a copolymer with a glass transition temperature $\left(T_{\mathrm{g}}\right)$ estimated by the Fox equation $\left(T_{\mathrm{g}, \text { Fox }}\right)^{43}$ of $82{ }^{\circ} \mathrm{C}$, therefore not allowing the preparation of nanocomposite films by water evaporation at ambient temperature. In the literature, comonomer compositions corresponding to copolymers with $T_{\mathrm{g}}$ above the polymerization temperature have been preferably used as they favor the encapsulation of the inorganic nanoparticles by decreasing chain mobility, thereby preventing possible migration of the clay platelets to the polymer/water interface in a thermodynamically driven process. ${ }^{8}$ Thus, the encapsulated morphology is aided by kinetics control as the inorganic nanoparticles are trapped inside a hard copolymer shell. With the intent to prepare film-forming latexes and verify the impact of the comonomer composition on the morphology of the hybrid particles, $\mathrm{R} 13$ and R14 with a MMA/BA ratio of $1 / 1$ (wt/wt), were prepared with MR7 and MR8 respectively (Table 2). The experimental conditions employed were similar to those that 
afforded stable latexes in R09 (MR7) and R12 (MR8) experiments, except that the relative amount of BA was increased resulting in a $T_{\mathrm{g}}$, Fox of $4{ }^{\circ} \mathrm{C}$. The cryo-TEM images of the prepared latexes are displayed in Figure 4.

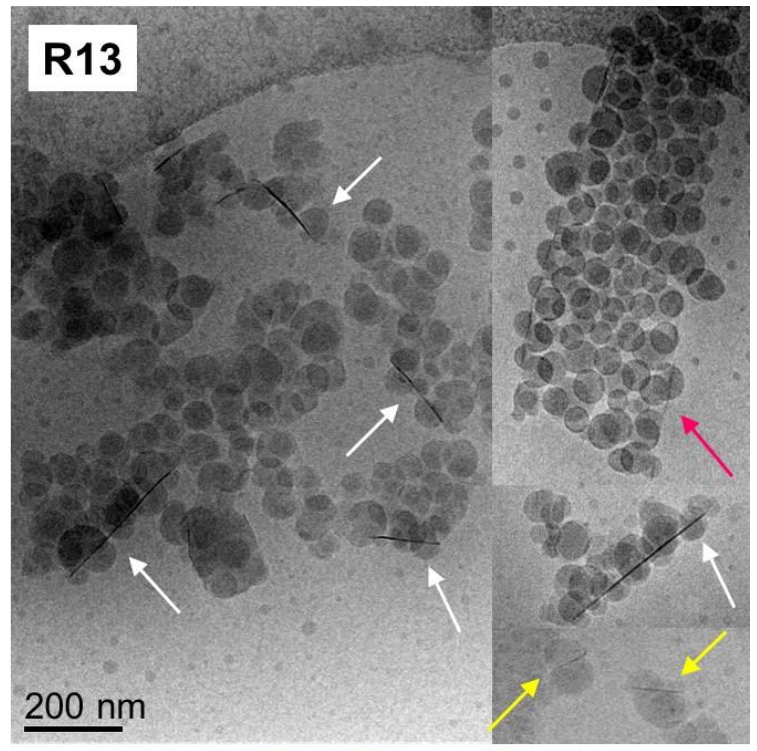

P(DMAEMA (26 $\left.^{-C O}-\mathrm{BA}_{20}\right)$

MR7, MMA/BA $=1 / 1 \mathrm{wt} / \mathrm{wt}$

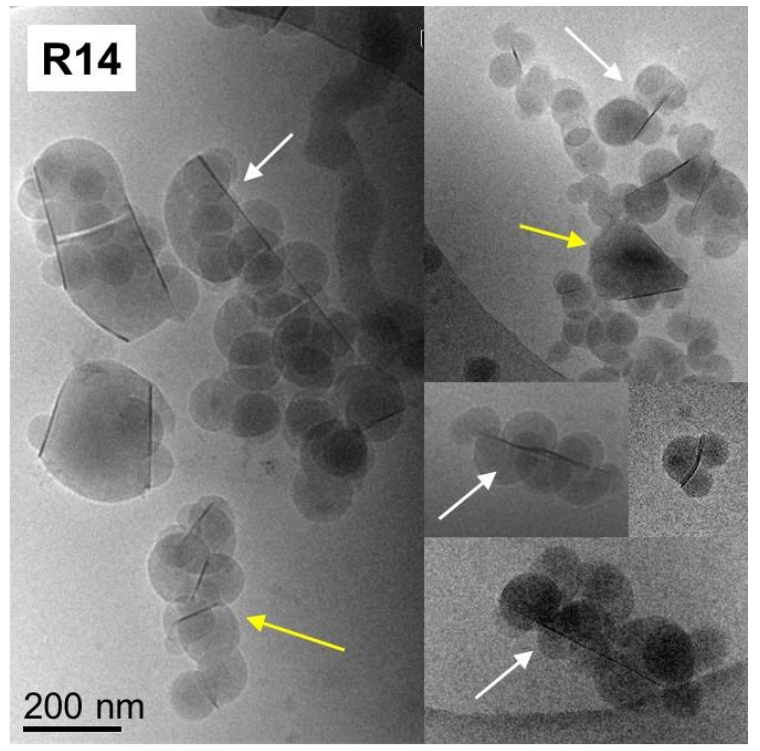

P(DMAEMA A $_{17}-$ Co-PEGA - -Co-BA 19 ) MR8, MMA/BA $=1 / 1 \mathrm{wt} / \mathrm{wt}$

Figure 4. Cryo-TEM images of $\mathrm{P}(\mathrm{MMA}-\mathrm{co}-\mathrm{BA}) / \mathrm{MMT}$ hybrid latex particles prepared with MMA/BA $=1 / 1 \mathrm{wt} / \mathrm{wt}$ using cationic macroRAFT agents at $\mathrm{pH} 5($ Monomer content $(\mathrm{Mon})=$ $10 \mathrm{wt} \%$, MMT = $5.0 \mathrm{~g} \mathrm{~L}^{-1}$ ). R13 (MR7) and R14 (MR8). See Table 2 for experimental conditions. White arrows point to MMT platelets decorated with polymer particles, pink arrows point to hybrid particles orthogonal to electron beam and yellow arrows point to MMT platelets sandwiched between polymer nodes.

The cryo-TEM micrographs of latex R13 synthesized with the P(DMAEMA $\left.26^{-}-\mathrm{D} o-\mathrm{BA}_{20}\right)$ (MR7) macroRAFT agent revealed MMT platelets decorated with polymer particles (indicated by white arrows in Figure 4), which did not cover the faces of the clay platelets homogeneously. However, the contours of the clay platelets were preserved. Some small clay platelets were completely sandwiched between polymer nodules (indicated by yellow arrows in Figure 4). The cryo-TEM images of latex R14 synthesized with MR8 also revealed polymer-decorated MMT platelets. However, in this sample, more sandwiched particles were 
found and some of them were agglomerated. Both film-forming hybrid latexes afforded transparent nanocomposite films by water evaporation at ambient temperature (Figure S8, Supporting Information). According to Pearson et al., ${ }^{34}$ the sandwiched morphology of LDHcontaining film-forming hybrid latexes synthesized by RAFT-mediated emulsion polymerization is presumably the equilibrium morphology, resulting from the polymerization temperature exceeding the $T_{\mathrm{g}}$ of the synthesized copolymer. In their work, the encapsulated morphology of film-forming hybrid latexes could be remarkably achieved using a lower molar mass macroRAFT agent $\left(M_{\mathrm{n}}=1850 \mathrm{~g} \mathrm{~mol}^{-1}\right)$, which was attributed to the increase in RAFT functions on the inorganic surface promoting more efficient polymer growth around the particles. However, in the present work using lower molar mass macroRAFT agents has shown to lead to lack of stability of MMT-containing hybrid latexes as discussed before and therefore another strategy is required for the preparation of MMT-containing film-forming hybrid latexes with encapsulated morphology.

\subsubsection{Towards Film Forming Hybrid Latexes with Encapsulated Morphology}

With the intent of encapsulating MMT platelets inside a film-forming copolymer shell, the syntheses of hybrid latexes were performed employing another strategy: a MMA-rich comonomer mixture $(\mathrm{MMA} / \mathrm{BA}=10 / 1 \mathrm{wt} / \mathrm{wt})$ was firstly fed to the reactor and another comonomer mixture $(\mathrm{MMA} / \mathrm{BA}=1 / 1 \mathrm{wt} / \mathrm{wt})$ was subsequently introduced. By doing so, we expected to kinetically trap the MMT platelets inside a hard copolymer shell and create a soft copolymer outer shell to enable film formation. Indeed, the film-forming properties of polymer latexes composed of hard core-soft shell nanoparticles have shown to be only influenced by the soft polymer shell. ${ }^{44}$ In addition, the final materials may display better 
mechanical properties than films prepared from latexes uniquely composed of low $T_{\mathrm{g}}$ (co)polymers due to the presence of high $T_{\mathrm{g}}$ polymer domains. ${ }^{45}$ It is worth reminding here that inorganic particles have been previously trapped inside a polymer shell by using a crosslinker during ATRP and RAFT-mediated emulsion polymerization, so polymer mobility around the inorganic particle would be reduced and the morphology could be fixed. ${ }^{19,34}$ However crosslinking of the polymer chains may affect interdiffusion during film formation, ${ }^{46,47}$ and eventually prevent film formation. ${ }^{34}$ In addition, the controlled character of the polymerization may also be affected. ${ }^{48}$

Hybrid latexes R15, R16 and R17 (Table 2) were prepared with MR8 employing this new strategy by varying the relative amounts of the two comonomer mixtures used while keeping the monomer concentration at $10 \mathrm{wt} \%$ (the final MMA/BA mass ratio of each sample is given in Table 2). The macroRAFT agent concentration used was $1.4 \mathrm{mmol} \mathrm{L}^{-1}$, which is slightly higher than the concentration previously used, so a higher final conversion could be reached using a lower initiator-to-macroRAFT agent molar ratio $([\mathrm{AIBA}] /[\mathrm{MR} 8]=0.3)$. The cryoTEM images of the samples are displayed in Figure 5. The morphology of latex R15 $(\mathrm{MMA} / \mathrm{BA}=1.3 \mathrm{wt} / \mathrm{wt})$, prepared with the lowest amount of MMA-rich comonomer mixture, was similar to the morphology of latex R14 (MMA/BA $\left.=1 / 1 \mathrm{wt} / \mathrm{wt}, T_{\mathrm{g}, \mathrm{Fox}}=4^{\circ} \mathrm{C}\right)$. As the amount of MMA-rich comonomer mixture was increased, the polymer coverage increased and particles completely covered with polymer could be identified in the cryo-micrographs of R17 (MMA/BA = $1.8 \mathrm{wt} / \mathrm{wt})$, although some polymer-decorated MMT platelets were also observed.

Another hybrid latex was prepared using a lower MMT concentration $\left(3.0 \mathrm{~g} \mathrm{~L}^{-1}\right)$ and a lower MR8 concentration to yield the same macroRAFT agent-to-MMT mass ratio of latex R17, while keeping the monomer concentration at $10 \%\left(\mathrm{R} 18, \mathrm{MR} 8=0.8 \mathrm{mmol} \mathrm{L}^{-1}\right.$, Table 2). The 
cryo-TEM images of this sample (Figure 5) reveals that as the MMT-to-monomer ratio was decreased, the polymer coverage increased leading to full clay encapsulation.

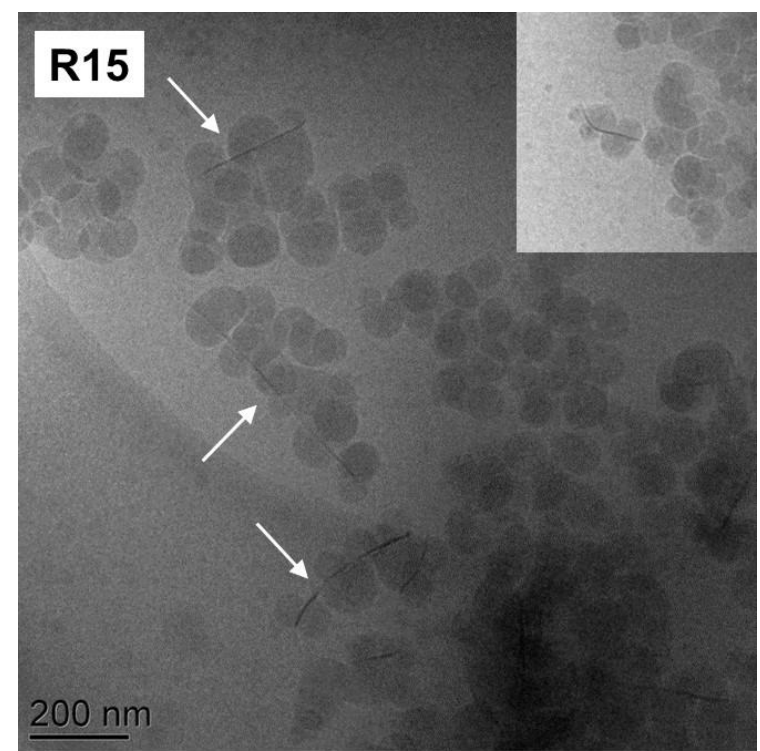

P(DMAEMA 17 -Co-PEGA - -Co-BA 19 ) MR8, $\mathrm{MMA} / \mathrm{BA}=1.3 \mathrm{wt} / \mathrm{wt}, \mathrm{MMT}=5 \mathrm{~g} \mathrm{~L}^{-1}$

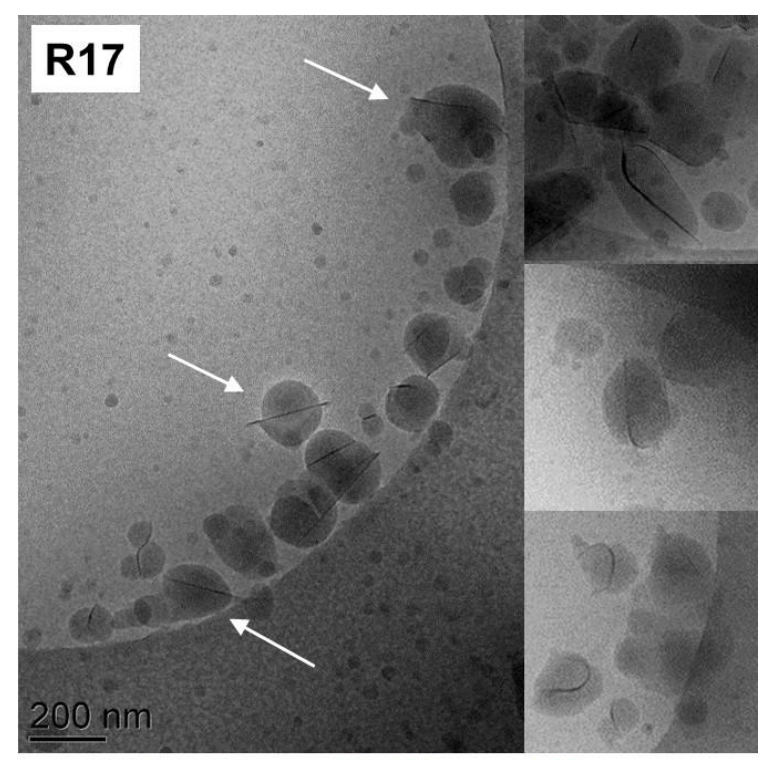

P(DMAEMA 17 -CO-PEGA - -CO-BA 19 ) MR8, $M M A / B A=1.8 \mathrm{wt} / \mathrm{wt}, \mathrm{MMT}=5 \mathrm{~g} \mathrm{~L}^{-1}$

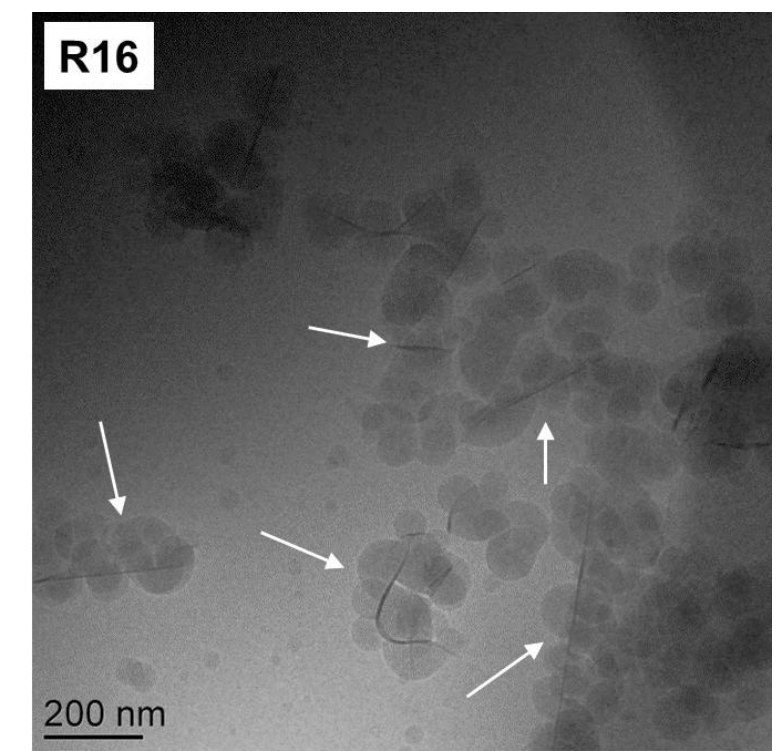

P(DMAEMA ${ }_{17}$-Co-PEGA - -Co-BA 19 ) MR8, $\mathrm{MMA} / \mathrm{BA}=1.4 \mathrm{wt} / \mathrm{wt}, \mathrm{MMT}=5 \mathrm{~g} \mathrm{~L}^{-1}$

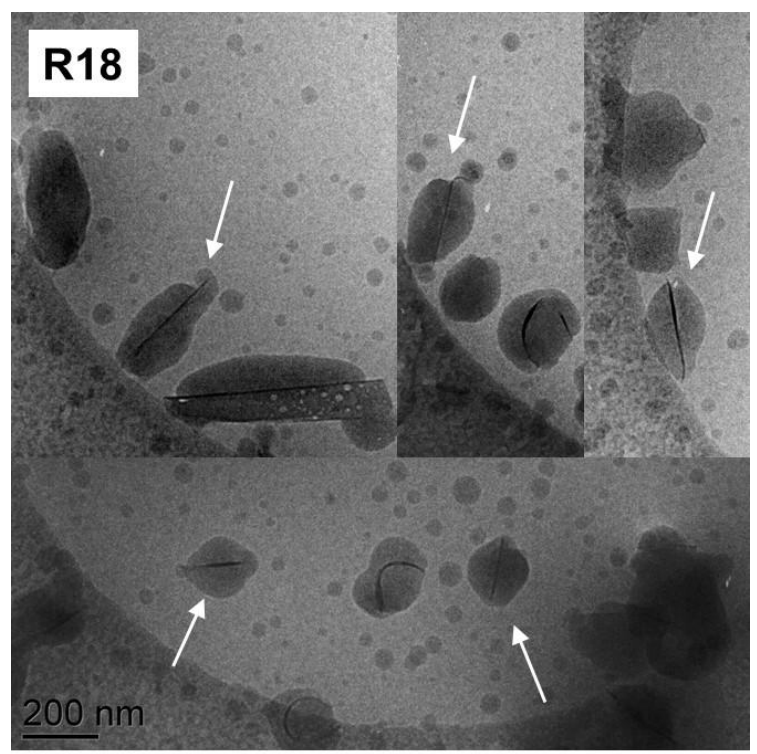

P(DMAEMA ${ }_{17}-$ Co-PEGA P $_{7}$-CO-BA 19 ) MR8, $\mathrm{MMA} / \mathrm{BA}=1.8 \mathrm{wt} / \mathrm{wt}, \mathrm{MMT}=3.0 \mathrm{~g} \mathrm{~L}^{-1}$

Figure 5. Cryo-TEM images of $\mathrm{P}(\mathrm{MMA}-\mathrm{co}-\mathrm{BA}) / \mathrm{MMT}$ hybrid latex particles prepared with P(DMAEMA ${ }_{17}-c o-P^{-}$DGA $\left._{7}-c o-\mathrm{BA}_{19}\right)$ (MR8) and two different monomer mixtures: MMA/BA $=10 / 1 \mathrm{wt} / \mathrm{wt}\left(m_{1}\right)$ and MMA/BA $=1 / 1 \mathrm{wt} / \mathrm{wt}\left(m_{2}\right)$ at $\mathrm{pH} 5$ (the total monomer concentration was $10 \mathrm{wt} \%) . \mathrm{R} 15\left(m_{2} / m_{1}=5, \mathrm{MMT}=5 \mathrm{~g} \mathrm{~L}^{-1}\right), \mathrm{R} 16\left(m_{2} / m_{1}=3, \mathrm{MMT}=5 \mathrm{~g} \mathrm{~L}\right.$ 
$\left.{ }^{1}\right), \operatorname{R} 17\left(m_{2} / m_{1}=2, \mathrm{MMT}=5 \mathrm{~g} \mathrm{~L}^{-1}\right)$ and $\mathrm{R} 12\left(m_{2} / m_{1}=2, \mathrm{MMT}=3 \mathrm{~g} \mathrm{~L}^{-1}\right)$. See Table 2 for experimental conditions. White arrows point to hybrid particles.

\subsection{Summary of Main Findings Regarding Parameters Influencing the Morphology of Hybrid Latexes}

This study has made it possible to assess the main relevant parameters influencing the morphology of MMT-containing hybrid latexes synthesized by RAFT-mediated emulsion polymerization. The first parameter is the nature of the macroRAFT agent. For the anionic ones, which displayed a relatively weaker interaction with the clay surface, the main morphology achieved was polymer-decorated MMT platelets. These macroRAFT agents also lead to the formation of a high amount of free polymer particles due the presence of free macroRAFT agent in the aqueous phase at the beginning of the polymerization. The molar mass of the macroRAFT agent has shown to be a key parameter influencing colloidal stability as macroRAFT agents with higher molar masses were necessary to prepare stable latexes with higher solid contents. These macroRAFT agents adsorb in such a conformation that enables more segments to be dangling into solution increasing colloidal stability. The interaction of these macroRAFT agents with the clay surface could be tuned by changing the $\mathrm{pH}$ from alkaline to acid, which influenced their ionization degree. However, as the interaction of the anionic macroRAFT agents with the clay surface could be increased, and thus polymerization was encouraged on the clay surface increasing polymer coverage and wettability, the stabilization potential of the macroRAFT agents decreased and eventually caused stability issues. These problems could be circumvented by using a macroRAFT agent with anionic repeating units grouped in a block which, despite the relatively higher number of anionic units, allowed it to adsorb more on the clay surface in such a conformation that favored the stabilization of the hybrid latexes (i.e., with the anionic block dangling into solution). 
However, free polymer particle was induced. Finally, the combination of a nonionic and an anionic macroRAFT agent also led to an increase in polymer/clay compatibility as the nonionic one displayed a higher interaction with the clay surface, being preferably absorbed while the anionic one would provide colloidal stability. For the cationic ones, which displayed a relatively stronger interaction with the clay surface and eventually adsorbed more on it, encapsulation was achieved. Again, macroRAFT agents with higher molar masses were required to stabilize the hybrid latexes. In this case, this parameter was also crucial for the stability of the initial macroRAFT agent-adsorbed MMT platelets, which was a concern and should be guaranteed. Another parameter that strongly affected colloidal stability was the presence of quaternized units in the macroRAFT agent structure as the quaternized cationic copolymers adsorbed in higher amounts and in a flatter conformation on the MMT surface compromising stability due to the absence of segments dangling into solution. As the polymerization was encouraged on the clay surface using cationic macroRAFT agents, less free polymer particles were formed in this case. The composition of the macroRAFT agent has also shown to be an important parameter. By using a cationic terpolymer comprising nonionic units, and thus displaying a stronger adsorption on the clay surface, a more homogeneous polymer coverage was achieved without compromising colloidal stability as the nonionic pendant chains provide additional steric stabilization. The morphology of the hybrid latexes is also influenced by thermodynamic factors associated to the polymer/clay interfacial energy. To assure encapsulation with a low $T_{\mathrm{g}}$ copolymer to yield film-forming latexes, it has been shown that a strategy consisting of two separate comonomer feedings is promising as a hard copolymer shell may be formed around the MMT platelets to kinetically trap them and avoid their migration to the polymer/water interface. Then a subsequent soft copolymer shell may be formed around the former to enable film formation. This way, hybrid latexes with encapsulated morphology and $T_{\mathrm{g}}$ lower than the reaction temperature could be prepared. 


\section{CONCLUSIONS}

Stable P(MMA-co-BA)/MMT hybrid latexes were successfully synthesized by surfactant-free starved-feed RAFT-mediated emulsion polymerization. Different types of macroRAFT agents were used and their compositions and structures were shown to strongly influence latex stability and morphology. In general, the use of fully ionized P(AA-co-PEGA-co-BA) macroRAFT agents in alkaline conditions resulted in the formation of polymer-decorated hybrid particles and induced secondary nucleation. By reducing the $\mathrm{pH}$ of the reaction medium, the affinity of the polymer phase for the clay surface increased but stability was impaired. The use of $\mathrm{PAA}_{41}-b-\mathrm{P}\left(\mathrm{PEGA}_{6}-c o-\mathrm{BA}_{4}\right)$ macroRAFT agent allowed the preparation of hybrid latexes with higher polymer content. Yet, the decorated morphology was again achieved. The nonionic $\mathrm{P}\left(\mathrm{PEGA}_{4}-\mathrm{co}-\mathrm{BA}_{3}\right)$, which displayed higher affinity for the MMT surface, when used concomitantly with $\mathrm{P}\left(\mathrm{AA}_{9}-c o-\mathrm{PEGA}_{9}-c o-\mathrm{BA}_{9}\right)$; resulted in a higher polymer coverage, although complete and uniform encapsulation was not achieved. In contrast, the use of cationic DMAEMA-based macroRAFT agents led to a different picture. Quaternized DMAEMA-based polymers could not stabilize the hybrid latexes due their strong interaction with the MMT surface causing them to adsorb flatter on it whereas $\mathrm{P}\left(\mathrm{DMAEMA}_{16}-\mathrm{co}-\mathrm{BA}_{15}\right), \mathrm{P}\left(\mathrm{DMAEMA}_{26}-c o-\mathrm{BA}_{20}\right)$ and $\mathrm{P}\left(\mathrm{DMAEMA}_{17}-c o-\mathrm{PEGA}_{7}-c o-\mathrm{BA}_{19}\right)$ led to the formation of a polymer shell on the MMT platelets preserving their shape anisotropy, while maintaining colloidal stability and minimizing at the same time the formation of free polymer particles.

Hybrid latexes synthesized using a film-forming comonomer feed composition led to thermodynamically favored morphologies such as MMT platelets decorated with polymer 
nodules as well as to sandwiched particles. By using another strategy, consisting of feeding two different comonomer mixtures to first create a hard polymer shell around the clay platelets and then an outer soft shell, polymer-encapsulated MMT platelets could be successfully prepared. This last strategy opens up the possibility of processing nanocomposite films with MMT platelets lying flat on the film substrate, which should create a tortuous path across the material, offering a great deal of promise for barrier coating applications.

\section{ASSOCIATED CONTENT}

\section{Supporting Information}

The Supporting Information is available free of charge on the ACS Publications. TEM analysis and particle size distribution of a typical MMT stock dispersion. Tables displaying experimental conditions that led to unstable latexes prepared with anionic, nonionic and cationic macroRAFT agents. Cryo-TEM images of latexes R02 and R03 displaying bare MMT platelets. Conversion versus time plots for the synthesis of hybrid latexes. Images of hybrid latexes SR7, R08, SR8, R09, R10, R11 and R12, prepared with cationic macroRAFT agents. Evolution of $Z_{\mathrm{av}}$ and poly values with conversion during the synthesis of latexes R08 and SR08. Images of nanocomposite films prepared with a film-forming composition.

\section{AUTHOR INFORMATION}

\section{Corresponding authors}

*E-mail: elodie.bourgeat-lami@univ-lyon1.fr (E. B-L.)

*E-mail: amsantos@usp.br (A. M. S.)

\section{Notes}

The authors declare no competing financial interest. 


\section{AKNOWLDEGMENTS}

This work was developed in the scope of the ENCIRCLE (Polymer-Encapsulation of Anisotropic Inorganic Particles by RAFT-Mediated Emulsion Polymerization) project enabled by IUPAC. The authors acknowledge São Paulo Research Foundation (FAPESP) (Brazil) (grant \#2010/50383-5 and grant \#2011/20533-8) and the National Center for Scientific Research (CNRS) (France) for financial support. Raíssa Silva Hardt (EEL/USP) is thanked for her help with the synthesis of hybrid latexes. Dr. Samuel Pearson is gratefully acknowledged for helpful discussions.

\section{REFERENCES}

1. Kotal, M.; Bhowmick, A. K., Polymer Nanocomposites from Modified Clays: Recent Advances and Challenges. Prog. Polym. Sci. 2015, 51, 127-187.

2. Ray, S. S., Recent Trends and Future Outlooks in the Field of Clay-Containing Polymer Nanocomposites. Macromol. Chem. Phys. 2014, 215, 1162-1179.

3. Chiu, C.-W.; Lin, J.-J., Self-Assembly Behavior of Polymer-Assisted Clays. Prog. Polym. Sci. 2012, 37, 406-444.

4. Sarkar, B.; Alexandridis, P., Block Copolymer-Nanoparticle Composites: Structure, Functional Properties, and Processing. Prog. Polym. Sci. 2015, 40, 33-62.

5. Bourgeat-Lami, E.; Lansalot, M., Organic/Inorganic Composite Latexes: The Marriage of Emulsion Polymerization and Inorganic Chemistry. Adv. Polym. Sci. 2010, 233, 53-123.

6. Faucheu, J.; Gauthier, C.; Chazeau, L.; Cavaille, J.-Y.; Mellon, V.; Bourgeat-Lami, E., Miniemulsion polymerization for synthesis of structured clay/polymer nanocomposites: Short review and recent advances. Polymer 2010, 51, 6-17.

7. Brunier, B.; Sheibat-Othman, N.; Chniguir, M.; Chevalier, Y.; Bourgeat-Lami, E., Investigation of Four Different Laponite Clays as Stabilizers in Pickering Emulsion Polymerization. Langmuir 2016, 32, 6046-6057. 
8. van Herk, A. M., Polymer Encapsulation of Single Clay Platelets by Emulsion Polymerization Approaches, Thermodynamic, and Kinetic Factors. Macromol. Reac. Eng. 2015, 10, 22-28.

9. Zengeni, E.; Hartmann, P. C.; Pasch, H., Encapsulation of Clay by Ad-Miniemulsion Polymerization: The Influence of Clay Size and Modifier Reactivity on Latex Morphology and Physical Properties. ACS Appl. Mater. Interfaces 2012, 4, 6956-6967.

10. Mičušík, M.; Bonnefond, A.; Reyes, Y.; Bogner, A.; Chazeau, L.; Plummer, C.; Paulis, M.; Leiza, J. R., Morphology of Polymer/Clay Latex Particles Synthesized by Miniemulsion Polymerization: Modeling and Experimental Results. Macromol. React. Eng. 2010, 4, 432-444.

11. Reyes, Y.; Paulis, M.; Leiza, J. R., Modeling the equilibrium morphology of nanodroplets in the presence of nanofillers. J. Colloid Interface Sci. 2010, 352, 359-365.

12. Reyes, Y.; Peruzzo, P. J.; Fernandez, M.; Paulis, M.; Leiza, J. R., Encapsulation of Clay within Polymer Particles in a High-Solids Content Aqueous Dispersion. Langmuir 2013, 29, 9849-9856.

13. Mballa, M. A. M.; Heuts, J. P. A.; van Herk, A. M., Encapsulation of non-chemically modified montmorillonite clay platelets via emulsion polymerization. Colloid Polym. Sci. 2013, 291, 501-513.

14. Zetterlund, P. B.; Thickett, S. C.; Perrier, S.; Bourgeat-Lami, E.; Lansalot, M., Controlled/Living Radical Polymerization in Dispersed Systems: An Update. Chem. Rev. 2015, 115, 9745-9800.

15. Bourgeat-Lami, E.; D’Agosto, F.; Lansalot, M., Synthesis of Nanocapsules and Polymer/Inorganic Nanoparticles Through Controlled Radical Polymerization At and Near Interfaces in Heterogeneous Media. Adv. Polym. Sci. 2016, 270, 123-161.

16. Qiao, X. G.; Dugas, P.-Y.; Charleux, B.; Lansalot, M.; Bourgeat-Lami, E., Synthesis of Multipod-like Silica/Polymer Latex Particles via Nitroxide-Mediated PolymerizationInduced Self-Assembly of Amphiphilic Block Copolymers. Macromolecules 2015, 48, 545556.

17. Qiao, X. G.; Lambert, O.; Taveau, J. C.; Dugas, P. Y.; Charleux, B.; Lansalot, M.; Bourgeat-Lami, E., Nitroxide-Mediated Polymerization-Induced Self-Assembly of Block Copolymers at the Surface of Silica Particles: Toward New Hybrid Morphologies. Macromolecules 2017, 50, 3796-3806.

18. Qiao, X. G.; Dugas, P. Y.; Charleux, B.; Lansalot, M.; Bourgeat-Lami, E., Nitroxide-Mediated Polymerization-Induced Self-Assembly of Amphiphilic Block 
Copolymers with a pH/Temperature Dual Sensitive Stabilizer Block. Polym. Chem. 2017, 8, 4014-4029.

19. Loiko, O. P.; Spoelstra, A. B.; van Herk, A. M.; Meuldijk, J.; Heuts, J. P. A., ATRP Mediated Encapsulation of Gibbsite: Fixation of the Morphology by Using a Cross-linker. Polym. Chem. 2017, 8, 2909-2912.

20. Loiko, O. P.; Spoelstra, A. B.; van Herk, A. M.; Meuldijk, J.; Heuts, J. P. A., An ATRP-Based Approach Towards Water-Borne Anisotropic Polymer-Gibbsite Nanocomposites. Polym. Chem. 2016, 7, 3383-3391.

21. Nguyen, D.; Zondanos, H. S.; Farrugia, J. M.; Serelis, A. K.; Such, C. H.; Hawkett, B. S., Pigment Encapsulation by Emulsion Polymerization Using Macro-RAFT Copolymers. Langmuir 2008, 24, 2140-2150.

22. Daigle, J.-C.; Claverie, J. P., A Simple Method for Forming Hybrid Core-Shell Nanoparticles Suspended in Water. J. Nanomater. 2008, 2008.

23. Ali, S. I.; Heuts, J. P. A.; Hawkett, B. S.; van Herk, A. M., Polymer Encapsulated Gibbsite Nanoparticles: Efficient Preparation of Anisotropic Composite Latex Particles by RAFT-Based Starved Feed Emulsion Polymerization. Langmuir 2009, 25, 10523-10533.

24. Das, P.; Zhong, W.; Claverie, J. P., Copolymer nanosphere encapsulated CdS quantum dots prepared by RAFT copolymerization: synthesis, characterization and mechanism of formation. Colloid Polym. Sci. 2011, 289, 1519-1533.

25. Zhong, W.; Zeuna, J. N.; Claverie, J. P., A versatile encapsulation method of noncovalently modified carbon nanotubes by RAFT polymerization. J. Polym. Sci. A: Polym. Chem. 2012, 50, 4403-4407.

26. Nguyen, D.; H., C.; Hawkett, B. S., Polymer coating of carboxylic acid functionalized multiwalled carbon nanotubes via reversible addition- fragmentation chain transfer mediated emulsion polymerization. J. Polym. Sci. A: Polym. Chem. 2013, 51, 250-257.

27. Zgheib, N.; Putaux, J.-L.; Thill, A.; Bourgeat-Lami, E.; D'Agosto, F.; Lansalot, M., Cerium oxide encapsulation by emulsion polymerization using hydrophilic macroRAFT agents. Polym. Chem. 2013, 4, 607-614.

28. Huynh, V. T.; Nguyen, D.; Such, C. H.; Hawkett, B. S., Polymer coating of graphene oxide via reversible addition-fragmentation chain transfer mediated emulsion polymerization. J. Polym. Sci. A: Polym. Chem. 2015, 53, 1413-1421.

29. Bourgeat-Lami, E.; França, A. J. P. G.; Chaparro, T. C.; Silva, R. D.; Dugas, P. Y.; Alves, G. M.; Santos, A. M., Synthesis of Polymer/Silica Hybrid Latexes by Surfactant-Free RAFT-Mediated Emulsion Polymerization. Macromolecules 2016, 49, 4431-4440. 
30. Nguyen, D.; Pham, B. T. T.; Huynh, V.; Kim, B. J.; Pham, N. T. H.; Bickley, S. A.; Jones, S. K.; Serelis, A.; Davey, T.; Such, C.; Hawkett, B. S., Monodispersed Polymer Encapsulated Superparamagnetic Iron Oxide Nanoparticles for Cell Labeling. Polymer 2016, $106,238-248$.

31. Li, K.; Dugas, P.-Y.; Bourgeat-Lami, E.; Lansalot, M., Polymer-encapsulated $\gamma$ Fe2O3 nanoparticles prepared via RAFT-mediated emulsion polymerization. Polymer 2016, 106, 249-260.

32. Perreira, A. C.; Pearson, S.; Kostadinova, D.; Leroux, F.; D'Agosto, F.; Lansalot, M.; Bourgeat-Lami, E.; Prevot, V., Nanocomposite Latexes Containing Layered Double Hydroxides via RAFT-Assisted Encapsulating Emulsion Polymerization. Polym. Chem. 2017, $8,1233-1243$.

33. Guimaraes, T. R.; Chaparro, T. d. C.; D'Agosto, F.; Lansalot, M.; dos Santos, A. M.; Bourgeat-Lami, E., Synthesis of multi-hollow clay-armored latexes by surfactant-free emulsion polymerization of styrene mediated by poly(ethylene oxide)-based macroRAFT/Laponite complexes. Polym. Chem. 2014, 5, 6611-6622.

34. Pearson, S.; Pavlovic, M.; Augé, T.; Torregrossa, V.; Szilagyi, I.; D’Agosto, F.; Lansalot, M.; Bourgeat-Lami, E.; Prévot, V., Controlling the Morphology of Film-Forming, Nanocomposite Latexes Containing Layered Double Hydroxide by RAFT-Mediated Emulsion Polymerization. Macromolecules 2018, 51, 3953-3966.

35. Dalmas, F.; Pearson, S.; Gary, B.; Chenal, J.-M.; Bourgeat-Lami, E.; Prevot, V.; Chazeau, L., Tailored Microstructure and Mechanical Properties of Nanocomposite Films Made from Polyacrylic/LDH Hybrid Latexes Synthesized by RAFT-Mediated Emulsion Polymerization. Polym. Chem. 2018, 9, 2590-2600.

36. Mballa Mballa, M. A.; Ali, S. I.; Heuts, J. P.; van Herk, A. M., Control of the Anisotropic Morphology of Latex Nanocomposites Containing Single Montmorillonite Clay Particles Prepared by Conventional and Reversible Addition- Rragmentation Chain Transfer Based Emulsion Polymerization. Polym. Int. 2012, 61, 861-865.

37. Silva, R. D.; Monteiro, I. S.; Chaparro, T. C.; Hardt, R. S.; Giudici, R.; BarrosTimmons, A.; Bourgeat-Lami, E.; Santos, A. M., Investigation of the Adsorption of Amphipathic macroRAFT Agents onto Montmorillonite Clay. Langmuir 2017, 33, 95989608.

38. Negrete-Herrera, N.; Putaux, J.-L.; David, L.; De Haas, F.; Bourgeat-Lami, E., Polymer/Laponite composite latexes: Particle morphology, film microstructure, and properties. Macromol. Rapid Commun. 2007, 28, 1567-1573. 
39. Bergaya, F.; Lagaly, G., General Introduction: Clays, Clay Minerals, and Clay Science. In Handbook of Clay Science, Bergaya, F.; Theng, B. K. G.; Lagaly, G., Eds. Elsevier: Amsterdam, 2006; Vol. 1, pp 1-18.

40. Cao, T.; Fasulo, P. D.; Rodgers, W. R., Investigation of the shear stress effect on montmorillonite platelet aspect ratio by atomic force microscopy. Appl. Clay Sci. 2010, 49, 21-28.

41. Tombacz, E.; Szekeres, M., Colloidal behavior of aqueous montmorillonite suspensions: the specific role of $\mathrm{pH}$ in the presence of indifferent electrolytes. Appl. Clay Sci. 2004, 27, 75-94.

42. Pavlovic, M.; Adok-Sipiczki, M.; Nardin, C.; Pearson, S.; Bourgeat-Lami, E.; Prevot, V.; Szilagyi, I., Effect of MacroRAFT Copolymer Adsorption on the Colloidal Stability of Layered Double Hydroxide Nanoparticles. Langmuir 2015, 31, 12609-12617.

43. Fox, T. G., Influence of diluent and of copolymer composition on the glass temperature of a polymer system. Bull. Am. Phys. Soc. 1956, 1, 123-132.

44. Bai, L.; Gu, J.; Huan, S.; Li, Z., Aqueous poly(vinyl acetate)-based core/shell emulsion: synthesis, morphology, properties and application. RSC Adv. 2014, 4, 2736327380.

45. Jiang, S.; Van Dyk, A.; Maurice, A.; Bohling, J.; Fasano, D.; Brownell, S., Design colloidal particle morphology and self-assembly for coating applications. Chem. Soc. Rev. 2017, 46, 3792-3807.

46. Zosel, A.; Ley, G., Influence of crosslinking on structure, mechanical properties, and strength of latex films. Macromolecules 1993, 26, 2222-2227.

47. Kessel, N.; Illsley, D. R.; Keddie, J. L., The diacetone acrylamide crosslinking reaction and its influence on the film formation of an acrylic latex. J. Coat. Technol. Res. 2008, 5, 285-297.

48. Wang, A. R.; Zhu, S., ESR Study on Diffusion-Controlled Atom Transfer Radical Polymerization of Methyl Methacrylate and Ethylene Glycol Dimethacrylate. Macromolecules 2002, 35, 9926-9933. 